\title{
MedienPädagogik
}

Zeitschrift für Theorie und Praxis der Medienbildung

\section{Weblogs in der Hochschullehre. Chancen und Herausforderungen}

\author{
Eine systematische Literaturanalyse des verpflichtenden und freiwilligen \\ studentischen Einsatzes von Weblogs
}

Alexandra Totter

\begin{abstract}
Zusammenfassung
Weblogs sind ein etabliertes Instrument in der Hochschullehre, das heutzutage in vielen Anwendungsbereichen zum Einsatz kommt. Mittlerweile gibt es eine Vielzahl empirischer Studien, die sich mit dem studentischen Einsatz von Weblogs in der Hochschullehre auseinandersetzen. Ziel dieser Literaturanalyse ist es, verpflichtendes und freiwilliges Bloggen von Studierenden im Rahmen ihrer universitären Ausbildung systematisch zu untersuchen. Dabei wird der Frage nachgegangen, welche Auseinandersetzung in empirischen Studien dazu erfolgt und welche Chancen und Herausforderungen sich bezüglich des verpflichtenden und freiwilligen studentischen Einsatzes von Weblogs in der Hochschullehre ergeben. Die ausgewählten Artikel werden dabei inhaltlich mittels eines induktiven Kategoriensystems analysiert. Chancen und Herausforderungen des jeweiligen Blogeinsatzes werden aufgezeigt und praktische Implikationen zusammengefasst. Anhand formaler Merkmale des Bloggens und des Studiums wird der studentische Blogeinsatz weiter charakterisiert. Abschliessend werden die Chancen und Herausforderungen gegenübergestellt und die in den empirischen Studien dargestellte theoretische Rahmung des studentischen Blogeinsatzes in einem mediendidaktischen Kontext erörtert.
\end{abstract}

Weblogs in Higher Education. Opportunities and Challenges. A systematic literature analysis of mandatory and voluntary student blogging

\begin{abstract}
Nowadays, weblogs are an established tool in higher education, and are being utilized in a wide field of applications. At the same time, one can find numerous empirical studies investigating student blogging in higher education. The aim of this literature review is to systematically investigate mandatory and voluntary student blogging in higher education. This paper considers the question of whether empirical studies discuss obligatory and voluntary student blogging and which opportunities and challenges arise in the context of the different use. Towards this goal, the content of the selected articles will be analysed using an inductive category system. Challenges and opportunities of the respective blog
\end{abstract}


use are pointed out and practical implications are summarized. Student blogging is further characterised by formal elements involved in the blogging activities and in the respective study curriculum. Finally, the challenges and opportunities of blogging will be compared, and the theoretical framing of the student blogging, as this occurs in the original empirical studies will be discussed in a media didactic context.

\section{Einleitung}

Mittlerweile sind Weblogs in der Hochschullehre eine feste Grösse und es gibt eine Vielzahl empirischer Untersuchungen, die sich mit dem studentischen Blogeinsatz auseinandersetzen (Deng und Yuen 2009a; O'Donnell 2006). Obwohl Weblogs auf Selbststeuerung und basisdemokratischen Prinzipien basieren, geht ihr Einsatz oftmals mit einer Leistungsbewertung und Selektion einher (Panke, Gaiser, und Maaß 2012). Studierende werden im Rahmen von Lehrveranstaltungen häufig dazu verpflichtet Weblogs zu führen, um einen entsprechenden Leistungsnachweis zu erhalten.

In dieser Verpflichtung ist Zwang präsent und man stösst auf ein Problem der Pädagogik, nämlich dem Verhältnis von Zwang und Freiheit (Plönges 2011, 152), das dem freiwilligen oder verpflichtenden studentische Blogeinsatz zugrunde liegt.

Beispielhaft werden im folgenden Exkurs zwei theoretische Positionen der Aufklärung und Gegenwart zum Verständnis von Zwang und Freiheit beleuchtet:

Bereits Immanuel Kant (1803) beschrieb dies als eines «der grössten Probleme der Erziehung [...], wie man die Unterwerfung unter den gesetzlichen Zwang mit der Fähigkeit sich seiner Freiheit zu bedienen, vereinigen könne» (A 33). Er begegnet dem Verhältnis von Zwang und Freiheit mit der Frage «Wie kultiviere ich die Freiheit bei dem Zwange?» (Kant 1803). Engelhardt und Seidl (2011) beschreiben Kants Verhältnis zum Begriff Zwang wie folgt: «Er bedient sich des Zwanges offenkundig, jedoch nicht in Ausübung zu Macht, sondern im Sinne eines positiven Faktors, um die Freiheit des Menschen zu kultivieren» (ebd., 119). Folgt man den Ausführungen Giesingers (2011) so nennt Kant

«drei Punkte, durch welche die Vereinbarkeit von Zwang und Freiheit sichergestellt werden soll. Erstens soll dem Kind [...] möglichst viel an Willkürfreiheit gewährt werden. Zweitens soll es lernen, dass man seine Zwecke nur erreichen kann, wenn es andere ihre Zwecke erreichen lässt. Drittens soll man dem Kind stets aufzeigen, dass der Zwang der Entwicklung seiner Freiheitsfähigkeit dient» (ebd., 262) ${ }^{1}$.

1 Gemäss Giesinger (2011) versteht Kant unter Willkürfreiheit «... Freiheit als Unabhängigkeit von eines anderen nötigender Willkür» (ebd., 260). 
Riefling (2013) schreibt, dass sich für Kant Zwang und Freiheit nicht ausschliessen müssen:

«Somit formuliert Kant in seiner Frage danach, wie man die Freiheit bei dem Zwange kultivieren könne, zwar das pädagogische Paradox, löst es jedoch dadurch auf, dass er Zwang und Freiheit prozessual aufeinander bezieht und miteinander verbindet» (ebd., 25).

Als theoretische Position der Gegenwart wird von einer Reihe von Autoren (Missomelius 2015; Volkers 2008; Maurer und Weber 2006) auf die Arbeiten von Michel Foucault verwiesen. Obwohl Foucault in erster Linie für seine Analytik der Macht bekannt ist, ist diese stets mit einer Analytik der Freiheit verbunden. In Foucaults Arbeiten sind Freiheit und Macht zwei kaum entwirrbare Wirklichkeiten, die als zwei gleichzeitige Erscheinungsweisen von Kräfteverhältnissen sichtbar werden (Mazumdar 2015). Seine Gouvermentalitätstheorie ermöglicht «den Blick auf Praktiken, in denen mit Hilfe von Subjektivierungstechnologien äußerer Zwang in Form von Machtstrukturen nach innen gekehrt und zu inneren Zwängen in der Konstitution des Subjekts wirksam wird» (Missomelius 2015, 159). Nach Maurer und Weber (2006) ersetzt Foucault «einfache Gegenüberstellungen von Zwang und Freiheit [...] durch einen reflexiven Modus von Regierung als Führen der Führungen» (ebd., 11). Foucault (1993) selbst schreibt:

«... governing people is not a way to force people to do what the governor wants; it is a versatile equilibrium, with complementarity and conflicts between techniques which assure coercion and processes through which the self is constructed or modified by himself» (ebd., 204).

Anhand dieser Positionen zeigt sich, dass Zwang und Freiheit nur auf den ersten Blick als binäre Gegensätze verstanden werden können. So fassen Fulda, Rosa und Thoma (2018) in ihrer Diagnose zusammen, dass sich beim genaueren Hinsehen offenbart, dass Zwang und Freiheit graduell und oft unmerklich ineinander übergehen können, und dass das eine in mancher Hinsicht ohne das andere nicht denkbar ist, oder dass Zwang und Freiheit «in einem [...] dialektischen Verhältnis stehen können, indem sie sich gegenseitig bedingen und steigern oder aber unterminieren» (ebd., 7).

So eindrücklich sich diese theoretischen Positionen mit dem Verhältnis von Zwang und Freiheit auseinandersetzen, so wird dabei deutlich, dass die Umsetzung in die pädagogische Praxis mit einer Reihe von Chancen und Herausforderungen verbunden ist. In diesem Beitrag bilden empirische Studien zum studentischen Blogeinsatz in der Hochschullehre die Grundlage für eine systematische Literaturanalyse, die untersucht, mit eben welchen Chancen und Herausforderungen ein freiwilliger und verpflichtender Blogeinsatz einher geht. 
Nach dem kurzen Exkurs zum Verhältnis von Zwang und Freiheit werden im Kapitel «Zum Einsatz von Weblogs in der Hochschullehre» die zentralen Eigenschaften und die Bedeutung von Weblogs im Hochschulkontext dargestellt. Die Fragestellung und das methodische Vorgehen werden in den anschliessenden Kapiteln vorgestellt. Im Kapitel «Ergebnisse» werden die inhaltliche Analyse und die Analyse der formalen Merkmale des Bloggens und des Studiums beschrieben. Abschliessend werden im Kapitel «Diskussion und Fazit» die Chancen und Herausforderungen des verpflichtenden und freiwilligen Blogeinsatzes einander gegenübergestellt und die in den empirischen Studien dargestellte theoretische Rahmung des studentischen Blogeinsatzes in einem mediendidaktischen Kontext diskutiert.

\section{Zum Einsatz von Weblogs in der Hochschullehre}

Weblogs werden als Teil sogenannter Web-2.0-Dienste verstanden. Jones (2015) charakterisiert diese wie folgt: «Web 2.0 places emphasis on user-generated content and participation, focused on the generation, manipulation and sharing of content» (ebd., 154). Obwohl sich Weblogs in ihren technischen Möglichkeiten und sozialen Praktiken ständig wandeln, lassen sie sich als regelmässig aktualisierte Webseiten beschreiben, deren Einträge chronologisch in umgekehrter Reihenfolge angeordnet sind, so dass der aktuellste Eintrag an erster Stelle erscheint (Walker 2005). Durch ihren öffentlichen Charakter ist es Leserinnen und Lesern möglich, zu einzelnen Beiträgen Kommentare und Notizen zu verfassen. Das tagebuchartige Element von Weblogs ist übrigens im Namen inkludiert. Der Begriff «Weblog» wurde von Jorn Barger 1997 als Kombination von «Web» und «Logbuch» geprägt (Blood 2000; Sim und Hew 2010). Ferner zeichnen sich Weblogs durch eine leichte Handhabung, kommunikativen Austausch und der Möglichkeit für direktes Feedback sowie den Aufbau von persönlichen Netzwerken aus (Panke, Gaiser, und Maaß 2012). Weblogs können als Einzelblog von nur einer Autorin oder einem Autor oder als Gruppenblog geführt werden, bei der eine beliebige Anzahl Autorinnen und Autoren Einträge verfasst. Sie sind meist öffentlich im Internet für jeden rezipierbar, können aber auch durch die Verwendung von Passwörtern für eine geschlossene Gruppe von Leserinnen und Lesern zugänglich gemacht werden.

Im praktischen Umgang mit Weblogs werden spezifische Begrifflichkeiten verwendet, bei denen es sich in erster Linie um technische Funktionen des WeblogSystems handelt. Ein Weblog enthält einzelne Einträge, sogenannte Blogposts oder kurz Posts, die in chronologisch umgekehrter Reihenfolge auf der Website gelistet werden; der zeitlich aktuellste Blogpost steht jeweils ganz oben. Die Elemente eines Blogposts können aus Texten, Bildern, Videos und Tonaufnahmen bestehen. Jeder Blogpost hat eine eigene permanente Internetadresse (Permalink), anhand derer man direkt auf den jeweiligen Blogpost zugreifen kann. So ist es möglich, bestimmte 
Blogposts anderer Weblogs direkt im eigenen Blog zu referenzieren. Jeder Blogpost kann einer definierten Kategorie zugeordnet werden und darüber hinaus durch Schlagworte (Tags) strukturiert werden. Unter einem «Thread» versteht man die Auflistung aufeinanderfolgender Blogposts zu einem bestimmten Thema über Kategorien oder Tags. Ein weiteres wichtiges Element von Weblogs ist die Funktion des Kommentierens. Leserinnen und Leser von Weblogs können zu den einzelnen Blogposts Kommentare schreiben. Diese können gleich veröffentlicht oder durch die Autorin oder den Autor moderiert werden (Appelt 2010; Buchem et al. 2011).

Mittlerweile sind Weblogs eine feste Grösse in der Hochschullehre und es gibt eine Vielzahl von Anwendungsbereichen: Sie werden als Lernjournale, Tagebücher, zur Dokumentation und Reflexion persönlicher Lernerfahrungen eingesetzt, dienen zur Interaktion, Kommunikation und Kooperation und werden in den Lehrveranstaltungen als Management- und Assessment-Tools genutzt (Sim und Hew 2010). Auch werden Weblogs eine Reihe von Potentialen zugeschrieben. So bieten sie gemäss Grell und Rau (2011) «... die Möglichkeit, das eigene Denken - durch die Verschriftlichung [...] - sichtbar und somit für Reflexionen und Rückmeldungen nutzbar zu machen» (ebd., 9). Weiter weisen die Autorin und der Autor darauf hin, dass der Prozess des «Sharings» Ausgangspunkt von Transformationsprozessen sein kann.

\section{Fragestellung}

Obwohl es mittlerweile eine Vielzahl empirischer Studien zum studentischen Bloggen gibt, findet sich in der Literatur noch keine systematische Analyse zum verpflichtenden und freiwilligen Einsatz von Weblogs.

Mit Hilfe einer systematischen Literaturanalyse sollen folgende Fragestellungen beantwortet werden:

- Welche Chancen und Herausforderungen werden in empirischen Studien zum verpflichtenden und freiwilligen studentischen Blogeinsatz geschildert?

- Welche formalen Merkmale des studentischen Blogeinsatzes und des Studiums finden sich in den empirischen Studien? Wie lässt sich das verpflichtende und freiwillige studentische Bloggen anhand dieser formalen Kriterien (weiter) charakterisieren?

\section{Methode}

Die systematische Literaturanalyse wurde in Anlehnung an die Richtlinien von Petticrew und Roberts (2006) durchgeführt. Dabei wurde eine gewisse Anzahl an Literaturdatenbanken ausgewählt und systematisch mittels vordefinierter Kombinationen von Suchbegriffen durchsucht. Anhand von Ein- und Ausschlusskriterien wurden die Ergebnisse der Suchabfrage weiter eingegrenzt. Beiträge, die den Kriterien entsprachen, wurden im Anschluss einer inhaltlichen Analyse unterzogen. 


\section{Auswahl der Literaturdatenbanken}

Da die Forschung zu Weblogs in unterschiedlichen Fachdisziplinen erfolgt, wurden folgende Literaturdatenbanken aus den Erziehungs- und Bildungswissenschaften, der Psychologie und der allgemeinen wissenschaftlichen Literatur ausgewählt: «ERIC» (https://eric.ed.gov), «FIS Bildung» (http://fis-bildung.de), «PsycINFO» (https://www.apa.org/pubs/databases/psycinfo/), «Clarivate Web of Science» (http:// www.webofknowledge.com) und «Google Scholar» (https://scholar.google.com).

\section{Formulierung der Suchabfragen}

In allen Datenbanken wurden folgende Operationen in der Freitextsuche verwendet: «*blog*»AND «studierend"»; «*blog*» AND «higher education». Für die Suche in Google Scholar wurde die Suchabfrage "*blog*", «higher education» und "*empirical $^{*} »$ verwendet. Bezüglich des Erscheinungsjahrs der Publikationen erfolgte keine Einschränkung. Die Suche erfolgte zwischen dem 10. und 17. Oktober 2016 und ergänzend bzw. aktualisierend am 14. Januar 2018.

\section{Ein- und Ausschlusskriterien}

Die Suchabfragen ergaben 699 unterschiedliche Treffer. Daraus wurden nur Artikel ausgewählt, bei denen es sich um

(a) empirische Studien oder Reviews empirischer Studien in

(b) deutscher oder englischer Sprache handelt, die in

(c) Zeitschriften, Büchern oder als Dissertationen publiziert sind. Die Nutzung bzw. der Einsatz von Weblogs musste

(d) im Hochschulkontext erfolgen und

(e) die Blogaktivität sollte von Studierenden durchgeführt werden.

(f) Schliesslich mussten die Artikel über den Verbund Schweizer Hochschulbibliotheken (und deren Fernleihe) zugänglich sein.

Nach Durchsicht der 699 Abstracts kamen 123 Beiträge in die engere Auswahl, die vollständig gelesen wurden. Den Ein- und Ausschlusskriterien entsprachen 98 Beiträge, die in einem nächsten Schritt inhaltlich analysiert wurden.

\section{Inhaltsanalyse der Beiträge}

Ausgangslage der inhaltlichen Analyse war der in den Beiträgen beschriebene studentische Blogeinsatz.

Um die erste Fragestellung zu beantworten, wurde in einem ersten Schritt unterschieden, ob verpflichtendes, freiwilliges oder beide Formen des studentischen Bloggens in den Beiträgen geschildert wurde. Beiträge, die keine Information dazu erhielten, wurden von der Inhaltsanalyse ausgeschlossen. 
Im Anschluss wurden die Beiträge in einem mehrstufigen Verfahren der Kategorienbildung und Codierung entlang der Hauptkategorien

1. mit dem Blogeinsatz verbundene Chancen,

2. Herausforderungen und

3. praktische Implikationen analysiert.

Unterkategorien wurden induktiv am Material entwickelt und ausdifferenziert. Ein Überblick über die relevanten Beiträge erfolgt tabellarisch. Die Ergebnisse der Inhaltsanalyse werden entlang der Hauptkategorien beschrieben.

Zur Beantwortung der zweiten Fragestellung wurden aus den Artikeln formale Merkmale des studentischen Bloggens und des Studiums extrahiert. Im Anschluss erfolgte die Analyse aller empirischer Artikel hinsichtlich der Ausprägungen dieser Merkmale. Diese Ergebnisse werden primär quantitativ dargestellt.

\section{Literaturanalyse}

Insgesamt wurden 98 Beiträge in die Literaturanalyse inkludiert, davon 94 empirische Studien ${ }^{2}$, die methodisch mehrheitlich schriftliche und mündliche Befragungen der Studierenden sowie Inhalts- und Frequenz-Analysen der Blogbeiträge und Kommentare der Studierenden einsetzten. Als weitere methodische Ansätze fanden sich in vier Artikel Soziale Netzwerkanalysen (SNA) (Fessakis, Tatsis, und Dimitracopoulou 2008; Pavo und Rodrigo 2015; Jimoyiannis und Tsiotakis 2017; Sharma und Tietjen 2016). Eine graphische Netzwerkanalyse wurde von Fessakis, Dimitracopoulou und Palaiodimos (2013) erstellt und Wang, Hou und Wu (2017) führten eine Sequenzanalyse (Lag Sequential Analysis, LSA) durch. Ein Artikel analysierte die Blogaktivitäten der Studierenden mittels diskursiver Psychologie (Lester und Paulus 2011).

\section{Ergebnisse Teil 1: Chancen und Herausforderungen des verpflichtenden und frei- willigen studentischen Blogeinsatzes}

Zur Beantwortung der Frage, mit welchen Chancen und Herausforderungen der studentischen Blogeinsatz einhergeht, konnten insgesamt elf Artikel identifiziert werden, die sich vertieft mit dieser Thematik auseinandersetzten. Dabei wurden in sechs Studien Weblogs verpflichtend eingesetzt, in zwei Studien war das Bloggen freiwillig, drei Studien verglichen die beiden Formen des studentischen Blogeinsatzes. Weiter fanden sich vier Literatur-Reviews zum Einsatz von Weblogs in der Hochschullehre (Deng und Yuen 2009a; Grell und Rau 2011; Schindler et al. 2017; Sim und Hew 2010), die diese Thematik diskutierten.

2 Die vollständige Übersicht der 94 analysierten Beiträge findet sich im Anhang., zwecks Lesbarkeit wurden diese mit Kürzel versehen. 
Im Folgenden werden die Ergebnisse gesondert nach verpflichtendem und freiwilligem studentischem Blogeinsatz dargestellt. Artikel, die freiwilliges und verpflichtendes Bloggen verglichen, sind gesondert in Tabelle 3 zusammengefasst. Die Ergebnisse der Analyse dieser Artikel wurden in die Kapitel «Verpflichtendes Bloggen» und «Freiwilliges Bloggen» integriert.

\section{Verpflichtendes Bloggen}

Wie aus Tabelle 1 hervorgeht, erfolgte der verpflichtende studentische Blogeinsatz in verschiedenen Stufen und Studienfächern. Auch variierte der Umfang, die Dauer der Blogaktivität und die Vorgaben, worüber gebloggt werden sollte.

\begin{tabular}{|c|c|c|}
\hline Studie & Blogging-Kontext \& Teilnehmende & Design \\
\hline $\begin{array}{l}\text { [D1] Dalgarno, } \\
\text { Reupert \& Bishop } \\
\text { (2015) }\end{array}$ & $\begin{array}{l}91 \text { Bachelor-Studierende der Primarlehrerbildung reflek- } \\
\text { tierten in mind. vier Blogposts \& vier Kommentaren über } \\
\text { Verhaltensmanagement während des fünf-wöchigen Be- } \\
\text { rufspraktikums. } \\
\text { Qualität der Blogposts wurde beurteilt. (Australien) }\end{array}$ & $\begin{array}{l}\text { Fragebogen, } \\
\text { Fokusgruppen, } \\
\text { Blog- \& Kom- } \\
\text { mentar-Frequenz }\end{array}$ \\
\hline $\begin{array}{l}\text { [F3] Freeman \& } \\
\text { Brett (2012) }\end{array}$ & $\begin{array}{l}\text { Zwölf Master- \& PhD-Studierende berichteten in mind. elf } \\
\text { Blogposts mit Hilfen von fünf themenspezifischen und zwei } \\
\text { freiwilligen Prompts über ihre Interessen und Erfahrun- } \\
\text { gen als graduierte Studierende während der 13-wöchigen } \\
\text { Lehrveranstaltung (LVA) eines Fernuniversitätslehrganges } \\
\text { zu Technologien und Lerntheorien in Erziehungswissen- } \\
\text { schaften. } \\
\text { Blogaktivität wurde von Studierenden mittels Beurtei- } \\
\text { lungsraster selbst beurteilt und machte } 23 \% \text { der Note aus. } \\
\text { (Kanada) }\end{array}$ & $\begin{array}{l}\text { Fragebogen, In- } \\
\text { haltsanalyse von } \\
\text { Studierenden- } \\
\text { kommentaren }\end{array}$ \\
\hline $\begin{array}{l}\text { [G3] Gebhardt \& } \\
\text { Jenert (2011) }\end{array}$ & $\begin{array}{l}52 \text { Studierende der Wirtschaftslehrpersonen-Ausbildung } \\
\text { dokumentierten und reflektierten anhand stark strukturier- } \\
\text { ter Reflexionsfragen und vorgegebener Deadlines die Um- } \\
\text { setzung eines Praxisprojektes und interagierten mit Peers } \\
\text { während der 13-wöchigen LVA zu aktuellen Problemen der } \\
\text { Wirtschaftsdidaktik. } \\
\text { Blogaktivität wurde von Dozierenden beurteilt und machte } \\
40 \% \text { der Leistungsbeurteilung aus. (Schweiz) }\end{array}$ & $\begin{array}{l}\text { Online-Fragebo- } \\
\text { gen, Inhaltsana- } \\
\text { lyse der Blog- } \\
\text { posts }\end{array}$ \\
\hline $\begin{array}{l}\text { [L2] Lester \& } \\
\text { Paulus (2011) }\end{array}$ & $\begin{array}{l}168 \text { Bachelor-Studierende eines Einführungskurses in } \\
\text { Ernährungswissenschaften reflektierten während zwei } \\
\text { Wochen zum Thema Nahrungsergänzungsmittel in mind. } \\
\text { einem Blogpost \& fünf Kommentaren vor und nochmals } \\
\text { nach Besuch der LVA was sie lernten und/oder wie sich ihr } \\
\text { Verständnis zu Nahrungsergänzungsmittel veränderte. } \\
\text { Blogaktivität war Teil der Mitarbeitsnote der Studierenden. } \\
\text { (USA) }\end{array}$ & $\begin{array}{l}\text { Inhaltsanalyse } \\
\text { der Blogposts } \\
\text { mittels diskursi- } \\
\text { ver Psychologie }\end{array}$ \\
\hline
\end{tabular}




\begin{tabular}{|l|l|l|}
\hline Studie & Blogging-Kontext \& Teilnehmende & Design \\
\hline $\begin{array}{l}\text { [S3] Sullivan } \\
\text { \& Longnecker } \\
\text { (2014) }\end{array}$ & $\begin{array}{l}\text { 62 Bachelor- \& Master-Studierende hatten in vier Wissen- } \\
\text { schaftskommunikations-LVAs die Möglichkeit Schreibpra- } \\
\text { xis zu sammeln, zu verstehen, wie akademische Theorien } \\
\text { für praktische Probleme genutzt werden können und Er- } \\
\text { fahrungen mit Peers zu teilen. In CSC- und SW-LVA verfass- } \\
\text { ten Studierende einen Blogpost \& drei Kommentare pro } \\
\text { Semester, in der S\&M-LVA zwei Blogposts pro Semester \& } \\
\text { zwei Kommentare wöchentlich, in der D\&E-LVA einen Blog- } \\
\text { post pro Semester und wöchentliche Kommentare. } \\
\text { Blogaktivität machte zwischen 10\% und 20\% des Leis- } \\
\text { tungsauftrages aus. (Australien) }\end{array}$ & $\begin{array}{l}\text { Fragebogen- } \\
\text { mentar-Frequenz }\end{array}$ \\
\hline [W4] Wolf (2010) & $\begin{array}{l}\text { 68 Public Relations Bachelor-Studierende reflektierten in } \\
\text { mind. vier Blogposts \& vier Kommentaren während des } \\
\text { letzten einsemestrigen Berufspraktikums über die Notwen- } \\
\text { digkeit einer kontinuierlichen Professionalisierung und } \\
\text { Verbesserung ihrer Informations-Literalität. } \\
\text { Blogaktivität macht 10\% der Leistungsbeurteilung aus. } \\
\text { (Australien) }\end{array}$ & $\begin{array}{l}\text { Online-Frage- } \\
\text { bogen, Blog- \& }\end{array}$ \\
Kommentar- \\
Frequenz
\end{tabular}

Tab. 1.: Beiträge mit ausschliesslich verpflichtendem Einsatz von Bloggen.

Die inhaltliche Analyse der Beiträge zu verpflichtendem Blogeinsatz zeigte folgende damit verbundene Chancen:

- Vorgaben und Prüfungsrelevanz: Genau definierte Themen und Vorgaben unterstützten Studierende beim Verfassen von Blogposts (Sullivan und Longnecker 2014). Minimalvorgaben (Wolf 2010) und Prüfungsrelevanz (Gebhardt und Jenert 2011) bewegten Studierende, Blogposts und Kommentare zu verfassen. In der vergleichenden Studie von Totter et al. (2016) bewerteten Studierende die verpflichtende Blogaktivität signifikant positiver als Studierende, bei denen die Blogaktivität nicht prüfungsrelevant war.

- Schreibfrequenz: In der Studie von Freeman und Brett (2012) zeigte sich, dass eine gewisse (verpflichtende und zeitlich regelmässige) Schreibfrequenz notwendig war, sodass Studierende ihre Weblogs als persönlichen Raum aufbauen konnten. Dadurch erhöhte sich die Wahrscheinlichkeit, dass Studierende zeitnah über Ideen und Themen mit einer für sie persönlichen und professionellen Resonanz schrieben.

Folgende Herausforderungen des verpflichtenden Blogeinsatzes fanden sich:

- Strikte Vorgaben und Verpflichtung: Studierende kritisierten die starke Strukturierung und die strikten Auflagen bezüglich Anzahl, Art und Deadlines zum Verfassen der Blogposts und Kommentare (Dalgarno, Reupert, und Bishop 2015; Gebhardt und Jenert 2011). Diese Kritik fand sich ebenfalls beim verpflichtenden Einsatz von Weblogs in der vergleichenden Studie von Chu, Chan, und Tiwari (2012). Verpflichtendes Kommentieren wurde von den Studierenden am negativsten 
bewertet und als belastend empfunden (Sullivan und Longnecker 2014). Insbesondere hoch-motivierte Studierende fühlten sich durch die genau definierten Themen und Vorgaben eingeschränkt (Sullivan und Longnecker 2014). Mit zunehmender Blogpraxis wurden Prompts, die von Studierenden verpflichtend bearbeitet werden mussten, eher als einengend empfunden (Freeman und Brett 2012).

- Hoher zeitlicher und inhaltlicher Aufwand: Die Blogaktivität wurde als anstrengend, unangenehm und belastend wahrgenommen (Gebhardt und Jenert 2011). Studierende kritisierten den hohen zeitlichen und inhaltlichen Aufwand der Blogaktivität, insbesondere während des Berufspraktikums (Dalgarno, Reupert, und Bishop 2015).

- Extrinsische Motivation: Ein Teil der Studierenden war extrinsisch motiviert (Gebhardt und Jenert 2011) und verfasste Blogposts primär, um die Lehrveranstaltung zu bestehen und gute Noten zu erhalten (Wolf 2010; Chu, Chan, und Tiwari 2012). Verpflichtendes Bloggen als Teil der akademischen Bildung wurde von einem Teil der Studierenden als Mittel zum Zweck, zum Erreichen eines Abschlusses wahrgenommen und weniger als Handlungsanleitung (Bending 2015).

- Besondere Textform: Lester und Paulus (2011) zeigten, dass das Verfassen von Blogposts von den Studierenden als «institutional talk» wahrgenommen wurde, bei denen sie sich an den Konventionen des «academic talk» orientierten. Dies stellte Studierende vor eine für sie widersprüchliche Interaktionsanforderung. Sie mussten auf diese Aufgabe in akzeptabler Art und Weise reagieren und sich gleichzeitig davor schützen, für ihr öffentlich dargestelltes Wissen verantwortlich gemacht zu werden. Studierende waren unsicher, ob ihre Blogaktivität der professionellen und Peergruppen-Norm entsprach (Bending 2015). Da es bislang noch keine Definition eines Blogging-Stils im akademischen Kontext gibt, war es für Studierende mitunter unklar, was von ihnen verlangt wurde (Sullivan und Longnecker 2014).

Als zentrale praktische Implikationen für den verpflichtenden Blogeinsatz nannten die Autoren folgende Aspekte:

- Leistungsbeurteilung und Rahmenbedingungen: Dalgarno, Reupert, und Bishop (2015) weisen darauf hin, dass durch die Art der Leistungsbeurteilung den Studierenden vermittelt werden soll, welche Aspekte der Blogaktivität, wie bewertet werden. Je nachdem, ob eine gewisse Anzahl und/oder eine bestimmte Qualität der Blogposts gefordert und bewertet wird, ergibt sich daraus ein unterschiedliches Bloggingverhalten der Studierenden. Sie verweisen auf die Bedeutung des «constructive alignment»; die intendierten Lernergebnisse, Lernaktivitäten und die Beurteilung müssen aufeinander abgeglichen sein. Gebhardt und Jenert (2011) sprechen in dem Zusammenhang von kollektiven «learning contracts». Sie und Sullivan und Longnecker (2014) schlagen vor die Blogging-Modalitäten und 
Beurteilungskriterien zu Beginn der Lehrveranstaltung mit den Studierenden zu diskutieren und so Verbindlichkeit bei gleichzeitig höherer Motivation zu Bloggen zu erreichen. Weiters wird angemerkt, dass für Studierende die öffentliche Darstellung ihres Wissens eine heikle Angelegenheit ist, die von Lehrpersonen mit Umsicht gesteuert werden muss (Lester und Paulus 2011).

- Minimalanforderungen und Flexibilität: Sullivan und Longnecker (2014) empfehlen wöchentliche Kommentare als Minimalanforderung für die Blogaktivität. Freeman und Brett (2012) schlagen vor, die Struktur und den Zeitplan von Prompts, wenn diese im Rahmen der Blogaktivität verwendet werden, zu verändern und im Laufe der Lehrveranstaltung schrittweise zu reduzieren.

\section{Freiwilliges Bloggen}

Es wurden zwei Studien analysiert, die ausschliesslich freiwilliges Bloggen thematisierten. Auch hier erfolgte der Blogeinsatz in verschiedenen Stufen und Studienfächern und die Dauer der Blogaktivität variierte (siehe Tabelle 2).

\begin{tabular}{|l|l|l|}
\hline Studie & Blogging-Kontext \& Teilnehmende & Design \\
\hline $\begin{array}{l}\text { [G1] Garcia, } \\
\text { Brown, \& Elbel- } \\
\text { tagi (2012) }\end{array}$ & $\begin{array}{l}\text { 32 Bachelor-Studierende der Hochschule für Kunst, Design } \\
\text { und Medien sammelten in Teams praktische Erfahrungen } \\
\text { zum auftragsorientieren Illustrieren. } \\
\text { Weblogs dienten während drei Wochen als Raum, in dem } \\
\text { Ideen, Recherchen und Probe-Entwürfe gesammelt und } \\
\text { Feedback gegeben werden konnte, bevor der jeweilige } \\
\text { Entwurf eingereicht wurde. (Grossbritannien) }\end{array}$ & $\begin{array}{l}\text { Analyse der } \\
\text { Illustrationen, } \\
\text { Blog- \& Kom- } \\
\text { mentarfrequenz } \\
\text { von drei Teams } \\
\text { Inhaltsanalyse } \\
\text { der Blogposts \& } \\
\text { Kommentare }\end{array}$ \\
\hline $\begin{array}{l}\text { Min2] Kerawalla, } \\
\text { \& Conole (2008) }\end{array}$ & $\begin{array}{l}\text { 108 Master-Studierende einer 20-wöchigen Online LVA } \\
\text { zum Thema «eLearning Professional» wurden gebeten } \\
\text { Weblogs auszuprobieren und darüber zu reflektieren. } \\
\text { (Grossbritannien). }\end{array}$ & $\begin{array}{l}\text { Teilstrukturierte } \\
\text { Interviews mit } \\
\text { 18 Studierenden } \\
\text { Inhaltsanalyse } \\
\text { der Blogposts }\end{array}$ \\
\hline
\end{tabular}

Tab. 2.: Beiträge mit ausschliesslich freiwilligem Einsatz von Bloggen.

Bei freiwilligem Bloggen zeigte die inhaltliche Analyse folgende damit verbundene Chancen:

- Diversität: Kerawalla et al. (2008) identifizierten in ihrer Studie anhand der freiwilligen Blogaktivität der Studierenden fünf verschiedene Blogging-Verhaltensweisen, die ganz unterschiedliche «learning needs» abdeckten:

1. Blogging-Verweigerung,

2. Ressourcen-Netzwerkbildung,

3. Unterstützungs-Netzwerkbildung,

4. Eigenständiges/autarkes Bloggen, 
5. besorgtes/unsicheres Bloggen, um den Anforderungen der Lehrveranstaltung gerecht zu werden.

Chu, Chan, und Tiwari (2012) betonten die Bedeutung des individuellen Engagements der Studierenden für die Blogaktivität (auch im Zusammenhang mit Gruppenblogs). Zu beachten ist dabei, dass alle vier Schlüsselaspekte des Bloggens Lesen, Schreiben, Kommentieren und Kommentare erhalten (Garcia, Brown, und Elbeltagi 2012) eine wichtige Rolle spielen.

- Persönliche Bedeutsamkeit: Gut durchdachte Blogposts, die einem persönlich bedeutsamen Beitrag entsprachen, fanden sich am ehesten im Zusammenhang mit Prompts, die freiwillig waren und bei denen kein Thema vorgegeben wurde (Freeman und Brett 2012).

Die Herausforderungen des freiwilligen Bloggens lassen sich wie folgt beschreiben:

- Extrinsische Motivation: Kerawalla et al. (2008) wiesen darauf hin, dass Studierende oft aufgabenfokussiert und ergebnisorientiert arbeiteten und es deshalb für sie schwierig sei, das Grundprinzip von Bloggen zu verstehen - nämlich Lernen durch die Entwicklung von Wissensgemeinschaften und «meaning making» sowie dem Teilen von Ressourcen und Meinungen zu unterstützen. Dieses fehlende Verständnis wurde auch in der Studie von Garcia, Brown, und Elbeltagi (2012) deutlich. Einige Teams zeigten eine geringe Blogbeteiligung. Studierende dieser Teams kritisierten, dass Peers die Beiträge so kurzfristig verfassten, dass keine Zeit mehr blieb, Verbesserungsvorschläge machen zu können (Garcia, Brown, und Elbeltagi 2012).

- Hoher zeitlicher Aufwand: Ähnlich wie in Kapitel «Verpflichtendes Bloggen» betonten die Studierenden auch hier den hohen zeitlichen Aufwand der Blogaktivität, insbesondere während des Berufspraktikums (Chu, Chan, und Tiwari 2012; Totter et al. 2016).

- Fehlende Vorgaben: Je grösser die Freiheit und Wahlmöglichkeit hinsichtlich Struktur, Inhalt und der zeitlichen Regulierung der Blogaktivität war, umso unwahrscheinlicher wurden vertiefte, kritische Reflexionen (Bending 2015).

Bezüglich der praktischen Implikationen aus den Erfahrungen des freiwilligen Bloggens sind sich die Autoren uneinig. Zum einen schlagen Garcia, Brown, und Elbeltagi (2012) vor, die Blogaktivität mit dem Leistungsauftrag zu verbinden, um künftig die Studierenden mehr zu motivieren, sich daran zu beteiligen, insbesondere dann, wenn die Aufgaben Zeit und reflexive Überlegungen erfordern. Zum anderen empfehlen Kerawalla et al. (2008) die Blogaktivitäten der Studierenden flexibel, freiwillig und relativ unstrukturiert zu gestalten, um den Studierenden die Möglichkeit zu geben, das Bloggen an ihre Bedürfnisse anzupassen und gleichzeitig die geforderten Aktivitäten zu erfüllen. 
In ihrer vergleichenden Studie weisen Totter et al. (2016) darauf hin, dass das Verfassen von Blogbeiträgen mit einen hohem Aufwand verbunden ist, der von Studierenden nicht zusätzlich zu ihren herkömmlichen Aufgaben und Anforderungen in Praktika ohne Weiteres zu leisten ist.

Zunächst abschliessend für den Ergebnisteil 1 finden sich in Tabelle 3 jene Artikel, die freiwilliges und verpflichtendes Bloggen verglichen. Unabhängig davon, ob Bloggen freiwillig oder verpflichtend war, verfassten Studierende in der Studie von Chu, Chan, und Tiwari (2012) regelmässig Blogeinträge. Die Autoren betonen, dass das pädagogische Design auf die Art der Blogaktivitäten einwirkt. Tätigkeiten, die viel mit Suchen und Finden von Information zu tun hatten, beeinflussten das Bloginteresse positiv.

\begin{tabular}{|c|c|c|}
\hline Studie & Blogging-Kontext \& Teilnehmende & Design \\
\hline $\begin{array}{l}\text { [B2] Bending } \\
(2015)\end{array}$ & $\begin{array}{l}\text { Sechs Bachelor-Studierende der Vorschul-Logopädie } \\
\text { konnten während ihrer berufspraktischen Ausbildung } \\
\text { Bloggen nutzen, um über ihre Erfahrungen zu reflektieren } \\
\text { als wesentliches Element ihrer Professionalisierung. } \\
\text { Studierende hatten zu Beginn ihres Studiums auch ver- } \\
\text { pflichtende Blogaktivitäten. (Grossbritannien) }\end{array}$ & $\begin{array}{l}\text { Unstrukturierte } \\
\text { Interviews }\end{array}$ \\
\hline $\begin{array}{l}\text { [C7] Chu, Chan, } \\
\text { \& Tiwari (2012) }\end{array}$ & $\begin{array}{l}53 \text { Bachelor-Studierende des Studiengangs Informations- } \\
\text { management (BScIM) reflektierten täglich während des } \\
\text { ein- bis drei-monatigem Berufspraktikums. } \\
\text { Blogaktivität macht 30\% der Praktikumsnote aus. } \\
\text { Während des zwölf-monatigen Berufspraktikums unter- } \\
\text { stützte Bloggen das Lehren und Lernen im klinischen } \\
\text { Praktikum von } 28 \text { Studierenden der Krankenpflege (BScN). } \\
\text { (Hong Kong) }\end{array}$ & $\begin{array}{l}\text { Inhaltsanalyse } \\
\text { der Blogposts } \\
\text { Telefoninter- } \\
\text { views } \\
\text { Analyse der Blog } \\
\text { \& Kommentar- } \\
\text { Frequenz } \\
\text { Stichprobenver- } \\
\text { gleiche hinsicht- } \\
\text { lich Blogging- } \\
\text { Frequenz, } \\
\text { subjektiver } \\
\text { Einschätzung \& } \\
\text { Lernprozesse }\end{array}$ \\
\hline $\begin{array}{l}\text { [T6] Totter et al. } \\
\text { (2016) }\end{array}$ & $\begin{array}{l}\text { Während des drei-wöchigen Berufspraktikums verfassten } \\
\text { Bachelor-Studierende der Primarlehrerbildung mit Hilfe } \\
\text { konkreter Schreibaufträgen täglich einen Blogpost zu } \\
\text { belastenden oder herausfordernden Situationen. } \\
\text { Die } 141 \text { Studierenden der Pädagogischen Hochschule } \\
\text { Zürich bloggten freiwillig. } \\
\text { Bei den } 141 \text { Studierenden der Pädagogischen Hochschule } \\
\text { Schwyz war Bloggen Teil des Leistungsauftrages des Prak- } \\
\text { tikums. (Schweiz) }\end{array}$ & $\begin{array}{l}\text { Online-Fragebo- } \\
\text { gen zu mehreren } \\
\text { Messzeitpunk- } \\
\text { ten }\end{array}$ \\
\hline
\end{tabular}

Tab. 3.: Beiträge, die freiwilliges und verpflichtendes Bloggen vergleichen. 
Ergebnisse Teil 2: Formale Merkmale des studentischen Blogeinsatzes und des Studiums

Nach der inhaltlichen Analyse der elf Artikel werden nun formale Merkmale des Bloggens und des Studiums dargestellt, anhand derer sich der studentische Blogeinsatz charakterisieren lässt. Die Ergebnisse basieren auf der Analyse aller 94 empirischer Studien.

Ein formales Merkmal des Blogeinsatzes war die Freiwilligkeit bzw. der Verpflichtungsgrad des Bloggens. Dieses Merkmal wurde anhand von Ausprägungen noch ausführlicher beschrieben. Als weitere Merkmale wurden ebenso Umfang, Dauer und Sozialform der Blogtätigkeit untersucht ${ }^{3}$. Merkmale des Studiums wurden ebenfalls systematisiert. Dabei wurde untersucht, auf welchen Stufen und in welchen Studienfächern der studentische Blogeinsatz erfolgte.

\section{Häufigkeitsverteilung von verpflichtendem und freiwilligem Bloggen ${ }^{4}$}

In 54 der 94 analysierten Artikel (57.4\%) wurde von den Autorinnen und Autoren ein verpflichtender studentischer Blogeinsatz geschildert. Freiwilliges Bloggen wurde in zwölf Beiträgen (12.8\%) beschrieben. In sechs Beiträgen (6.4\%) wurde beides, freiwilliges und verpflichtendes Bloggen eingesetzt bzw. verglichen. Keine Angaben dazu fanden sich in 22 Artikel (23.4\%).

\section{Zum Grad und Umfang der Verpflichtung}

Bei der Analyse der Artikel zeigte sich weiter, dass Verpflichtung nicht gleich Verpflichtung ist. So konnte eine Vielzahl unterschiedlicher Blog-Verpflichtungen identifiziert werden. In 16 der 54 Beiträge wurde Bloggen unspezifisch als «Teil der Leistungsbeurteilung» genannt. In den anderen Beiträgen wurde die Blogaktivität der Studierenden konkret beschrieben. Diese konnte zwischen 0.2\% (im Sinne eines Extrakredits) bis hin zu 60\% der Gesamtnote ausmachen. Die Blogaktivität war aber auch Teil der Mitarbeitsnote, Projektnote, Hausaufgabennote oder der Praktikumsanforderung. Ebenfalls wurde in einigen Artikeln beschrieben, dass nicht alle, sondern nur ausgewählte Blogbeiträge benotet wurden. Eine Übersicht findet sich in Tabelle 4.

3 Neben den genannten Merkmalen liessen sich noch weitere unterscheiden, die aber hier nicht berücksichtigt wurden: Zum Beispiel «Zugänglichkeit» - handelt es sich um öffentliche oder private Blogs, oder «Kommentarfunktion» - der Möglichkeit, Kommentare zum Blogpost zu verfassen.

4 Die vollständige Übersicht der 95 analysierten Beiträge findet sich im Anhang. Zwecks Lesbarkeit werden anstatt der Referenzen in den Tabellen Kürzel verwendet. 


\begin{tabular}{|l|l|}
\hline Grad der Verpflichtung & Artikel \\
\hline Blogaktivität < 10\% der Gesamtnote & D5, D8, I2, J1, Y1 \\
\hline $\begin{array}{l}\text { Blogaktivität zwischen } 10 \% \text { und 20\% der Ge- } \\
\text { samtnote }\end{array}$ & A1, D2, H4, H8, M4, N3, O2, S3, T1, W4, X1 \\
\hline $\begin{array}{l}\text { Blogaktivität zwischen 21\% und 30\% der Ge- } \\
\text { samtnote }\end{array}$ & B5, C7, F3, M1, R3, S1 \\
\hline $\begin{array}{l}\text { Blogaktivität zwischen 31\% und 40\% der Ge- } \\
\text { samtnote }\end{array}$ & G3, J3 \\
\hline Blogaktivität > 40\% der Gesamtnote & S1, S2 \\
\hline $\begin{array}{l}\text { Benotung (ausgewählte Arbeiten, finale Versio- } \\
\text { nen, 2x pro Semester, ect.) }\end{array}$ & $\mathrm{H} 3, \mathrm{~J} 2, \mathrm{M} 2, \mathrm{~N} 4, \mathrm{P} 3, \mathrm{R} 4, \mathrm{~W} 3$ \\
\hline Teil der Mitarbeitsnote & $\mathrm{B} 1, \mathrm{~L} 2$ \\
\hline Teil der Projektnote & $\mathrm{F} 2, \mathrm{~W} 1$ \\
\hline Teil der Praktikumsanforderung & $\mathrm{C} 8, \mathrm{C} 10$ \\
\hline Teil der Hausaufgabennote & $\mathrm{T} 2$ \\
\hline
\end{tabular}

Tab. 4.: Verpflichtungsgrad.

Der Umfang der Verpflichtung variierte ebenfalls. Sofern die Autorinnen und Autoren Aussagen zum Blogumfang machten (45 Artikel), wurde am häufigsten von den Studierenden verlangt, wöchentlich einen Blogpost zu verfassen. Das Verfassen von insgesamt vier Blogposts erschien den Autorinnen und Autoren ebenfalls angemessen (siehe Tabelle 5).

\begin{tabular}{|l|l|}
\hline Umfang & Artikel \\
\hline Täglicher Blogpost & C7, C8, G4, T6 \\
\hline Wöchentlicher Blogpost & $\begin{array}{l}\text { A1, A3, B2, C10, D6, H1, H4, H6, O2, P4, R5, S1, } \\
\text { S3, T4, X1 }\end{array}$ \\
\hline 2x pro Woche & H2, S4 \\
\hline 1 Blogpost & K3, L2, N1, S3 \\
\hline 2 Blogposts & B4, C2, D5, L1, M4 T5 \\
\hline 3 Blogposts & B5, F2, K1, N4 \\
\hline 4 Blogposts & B1, D1, J1, L4, P1, R1, T2, W1, W4 \\
\hline 5, 6, oder 7 Blogposts & F3, N3 \\
\hline
\end{tabular}

Tab. 5.: Umfang der Blogtätigkeit.

\section{Zur Dauer der Blogaktivität}

Die Dauer der Blogaktivität variierte zwischen zwei und 40 Wochen (37 Artikel nannten Details dazu). In vielen Artikeln (siehe Tabelle 6) wurde die Blogaktivität über ein Semester (zwischen zwölf und 16 Wochen) hinweg geführt (16 Artikel). 


\begin{tabular}{|l|l|}
\hline Dauer der Blogtätigkeit & Artikel \\
\hline < Semester (zwischen 2-9 Wochen) & $\begin{array}{l}\text { C5, C10, D6, G1, G2, G4, H1, J1, H2, L2, O1, O2, } \\
\text { P2, R4, W1, T6 }\end{array}$ \\
\hline Semester (12-16 Wochen) & $\begin{array}{l}\text { B3, B5, C7, C8, H7, J3, M1, M3, N3, P3, R2, S3, } \\
\text { S4, T2, T3, W3 }\end{array}$ \\
\hline$>$ Semester (bis zu 40 Wochen) & $\mathrm{C} 7, \mathrm{G} 5, \mathrm{H} 5, \mathrm{~K} 2, \mathrm{~L} 3, \mathrm{Y} 1$ \\
\hline
\end{tabular}

Tab. 6.: Dauer der Blogtätigkeit.

Sozialform des Bloggens - zum Einsatz von individuellen Weblogs, Gruppen- und Kursblogs

Als weiteres Merkmal der Blogtätigkeit liess sich unterscheiden, ob Studierende einen individuellen Weblog führten, oder ob sie ihre Blogposts in Gruppenblogs verfassten, entweder in mehreren Kleingruppen oder einem Klassen-/Kursblog. Wie die Ergebnisse in Tabelle 7 zeigen, gab es neben der ausschliesslichen Nutzung von individuellen Blogs auch Varianten. Einzelblogs wurden in Kombination mit einem Kurs/ Klassenblog oder mit Kleingruppenblogs geführt. Mehrere Studierende verfassten dabei Blogposts und Kommentare in einem Weblog. In dreizehn der 94 Artikel machten die Autorinnen und Autoren keine Angaben zu den verwendeten Sozialformen.

\begin{tabular}{|l|l|}
\hline Sozialformen & Artikel \\
\hline Individuelle Blogs & B4, B5, C6, C8, G4, H4, H5, I1, J2, K3, M1, O1, \\
& P4, R2, R5, S4, T3, T6, W3, X1, \\
\hline Individuelle Blogs + ein Kurs/Klassenblog & B3, C4, C9, D5, K1, N4, S1, T1, Y1 \\
\hline Individuelle Blogs + Kleingruppenblogs & A4, I3 \\
\hline Kleingruppenblogs & $\begin{array}{l}\text { A1, B1, C1, C2, C3, D3, D4, D7, D8, F1, F2, G1, } \\
\text { G2, G3, H1, H2, H3, H8, J1, J3, J4, K4, L1, L2, L3, } \\
\text { L5, M3, M4, N1, N2, N3, O2, P2, R1, R3, R4, S2, } \\
\text { T4, T5, W1, W4 }\end{array}$ \\
\hline Kleingruppenblogs + ein Kurs/Klassenblog & A3, D2, G5, M2, P1, S3 \\
\hline Ein Kurs/Klassenblog & A2, C1, D6, P3 \\
\hline
\end{tabular}

Tab. 7.: Sozialform des Bloggens.

Dichotomisiert man die Ausprägungen der Sozialformen in die zwei Kategorien individueller Blog und Gruppenblog, so zeigt sich, dass unabhängig vom Verpflichtungsgrad - verpflichtend oder freiwillig - in der Hochschullehre am häufigsten Gruppenblogs eingesetzt werden (siehe Tabelle 8). 


\begin{tabular}{|l|l|l|l|}
\hline & Individueller Blog & Gruppenblog & Gesamt \\
\hline Freiwillig & 6 & 9 & 15 \\
\hline Verpflichtend & 20 & 34 & 54 \\
\hline Gesamt & 26 & 43 & 69 \\
\hline
\end{tabular}

Tab. 8.: Häufigkeitsverteilung aufgeteilt nach Sozialform und Verpflichtungsgrad des Bloggens.

\section{Zum Einsatz von Weblogs in verschiedenen Stufen und Studienfächern}

In 52 Artikel wurde auf Bachelorstufe gebloggt. Zu Bloggen auf Masterstufe oder höher fanden sich 17 Artikel und in neun Artikel wurde auf mehreren Stufen gebloggt. In 16 der 95 Artikel machten die Autorinnen und Autoren keine Angaben dazu (siehe Tabelle 9).

\begin{tabular}{|l|l|}
\hline Stufen & Artikel \\
\hline Bachelorstufe & A4, B2, B3, B5, C1, C2, C3, C4, C5, C7, C8, D1, \\
& D2, D3, D5, D6, D8, F1, G1, G2, G4, G5, H1, H3, \\
& H4, H6, H7, I1, I2, J1, J2, J4, L1, L2, L3, L4, L5, \\
& M2, M4, N3, P1, P2, R4, R5, T1, T4, T5, T6, W1, \\
& W3, W4, X1 \\
\hline Masterstufe oder höher & A3, B4, C9, F3, H5, H8, K1, K2, K3, M1, O2, R1, \\
& R2, S1, S3, S4, T3 \\
\hline auf mehreren Stufen & A1, A2, D7, H2, I3, N1, N2, W2, Y1 \\
\hline
\end{tabular}

Tab. 9.: Einsatz von Blogs in verschiedenen Stufen.

Daten zum Verpflichtungsgrad und Stufen wurden ebenfalls zu dichotomen Variablen zusammengefasst (vgl. Tabelle 10) ${ }^{5}$. Dabei zeigte sich, dass auf Masterstufe oder höher, der studentische Blogeinsatz etwas häufiger freiwillig erfolgte als auf Bachelorstufe. Bei einer statistischen Prüfung mittels $\mathrm{Chi}^{2}$-Test zeigte sich allerdings kein statistisch signifikanter Unterschied $\left(\mathrm{Chi}^{2}(1)=1.118 \mathrm{p}=.290\right)$.

\begin{tabular}{|l|l|l|l|}
\hline & Bachelor & Master und höher & Gesamt \\
\hline Freiwillig & 10 & 5 & 15 \\
\hline Verpflichtend & 36 & 9 & 45 \\
\hline Gesamt & 46 & 14 & 60 \\
\hline
\end{tabular}

Tab. 10.: Verpflichtungsgrad des Bloggens aufgeteilt nach Stufen.

5 Dabei wurden Artikel, die beides, sowohl freiwilliges als auch verpflichtendes Bloggen beschrieben, jeweils als eigener Fall analysiert. Artikel, die über den Einsatz von Blogs auf mehreren Stufen berichteten, wurden ausgeschlossen. 
Die Ergebnisse zum Einsatz von Blogs in verschiedenen Studienfächern (siehe Tabelle 11) deckten sich grösstenteils mit den Resultaten von Sim und Hew (2010). Am häufigsten wurden Blogs im «Bildungsbereich» (Lehrerbildung, Erziehungswissenschaften, Fachdidaktik; 32 Artikel) eingesetzt, gefolgt von Studienfächern im Bereich «Informations- \& Naturwissenschaften» (Informatik, Naturwissenschaften, Gesundheit, Politikwissenschaften; 30 Artikel). Eine Zunahme des Blogeinsatzes zeigte sich in den Studienfächern «Sprache \& Kommunikation». Dort gab es einen Anstieg von $4 \%$ auf $14.9 \%$ (15 Artikel) und im Bereich «Wirtschaft» von 8\% auf $13.8 \%$ (13 Artikel) im Vergleich zur Untersuchung von Sim und Hew (2010).

\begin{tabular}{|l|l|}
\hline Studienfach & Artikel \\
\hline Bildungsbereich & A2, C1, C10, C6, D1, D3, D4, D6, F1, F3, G3, G4, \\
& G5, H2, H5, I3, M1, M2, N1, N2, N3, P1, P2, P4, \\
& R5, S1, S2, S4, T1, T4, T5, T6 \\
\hline Informations- \& Naturwissenschaften & A3, B2, B5, C4, C5, C7, C8, C9, D8, F2, H1, H7, I1, \\
& $\begin{array}{l}\text { 2, J4, K1, K4, L1, L2, M4, O1, R1, R2, R3, R4, T2, } \\
\text { T3, W3, X1, Y1 }\end{array}$ \\
\hline Sprache \& Kommunikation & A1, B4, C3, D2, D5, H3, H4, H6, L3, L4, L5, M3, \\
& N4, S3, W1 \\
\hline Wirtschaft & A4, B1, C2, D7, H8, J1, J2, J3, K2, K3, O2, W2, \\
& W4 \\
\hline Sonstiges & B3, G1, G2, H3, P3 \\
\hline
\end{tabular}

Tab. 11.: Bloggen in verschiedenen Studienfächern.

Unterscheidet man in den Studienfächern zwischen freiwilligem und verpflichtendem studentischem Bloggen (siehe Tabelle 12$)^{6}$, so fand sich im Studienfach «Sprache \& Kommunikation» nur einer von zwölf Artikeln, der freiwilliges Bloggen der Studierenden schilderte. Im Gegenteil dazu wurde im Studienfach «Informations- \& Naturwissenschaften» vergleichsweise häufig auch freiwilliges Bloggen eingesetzt. Die statistische Prüfung mittels $\mathrm{Chi}^{2}$-Test zeigte allerdings keinen statistisch signifikanter Unterschied $\left(\mathrm{Chi}^{2}(3)=2.157 p=.540\right.$,).

\begin{tabular}{|l|l|l|l|l|l|}
\hline & $\begin{array}{l}\text { Sprache \& } \\
\text { Kommunika- } \\
\text { tion }\end{array}$ & $\begin{array}{l}\text { Bildungsbe- } \\
\text { reich }\end{array}$ & $\begin{array}{l}\text { Informations- } \\
\text { \& Naturwis- } \\
\text { senschaften }\end{array}$ & Wirtschaft & Gesamt \\
\hline Freiwillig & 1 & 5 & 6 & 3 & 15 \\
\hline Verpflichtend & 12 & 22 & 16 & 9 & 59 \\
\hline Gesamt & 13 & 27 & 22 & 12 & 74 \\
\hline
\end{tabular}

Tab. 12.: Verpflichtungsgrad des Bloggens aufgeteilt nach Studienfächern.

6 Dabei wurden Artikel, die sowohl freiwilliges als auch verpflichtendes Bloggen beschreiben, jeweils als eigener Fall analysiert. Sonstige Studienfächer wurden von der Analyse ausgeschlossen. 
Ergänzend zur qualitativ orientierten inhaltlichen Analyse der Artikel, ermöglichte die Analyse der formalen Merkmale eine weitere Charakterisierung des Einsatzes von Weblogs in der Hochschullehre. Die Ergebnisse werden nun in Bezug auf die Fragestellungen diskutiert.

\section{Diskussion und Fazit}

In diesem Beitrag wurde eine systematische Literaturanalyse vorgestellt, die untersucht, wie in empirischen Studien die Auseinandersetzung mit verpflichtendem und freiwilligem studentischem Bloggen erfolgt. Die inhaltliche Analyse der inkludierten Artikel identifizierte eine Reihe von damit verbundenen Herausforderungen und Chancen. In Tabelle 13 werden diese Herausforderungen und Chancen des verpflichtenden und freiwilligen studentischen Blogeinsatzes gegenübergestellt und im Anschluss diskutiert.

\begin{tabular}{|l|l|l|}
\hline & Verpflichtendes Bloggen & Freiwilliges Bloggen \\
\hline $\begin{array}{l}\text { Herausforde- } \\
\text { rungen }\end{array}$ & $\begin{array}{l}\text { - hoher zeitlicher \& inhaltlicher Auf- } \\
\text { wand } \\
\text { - extrinsische Motivation } \\
\text { - strikte Vorgaben \& Verpflichtung } \\
\text { - besondere Textform }\end{array}$ & $\begin{array}{l}\text { - hoher zeitlicher Aufwand } \\
\text { - extrinsische Motivation }\end{array}$ \\
\hline Chancen & $\begin{array}{l}\text { - Vorgaben \& Prüfungsrelevanz Vorgaben } \\
\text { - Schreibfrequenz }\end{array}$ & $\begin{array}{l}\text { - Diversität } \\
\text { - persönliche Bedeutsamkeit }\end{array}$ \\
\hline
\end{tabular}

Tab. 13.: Herausforderungen und Chancen des verpflichtenden und freiwilligen studentischen Bloggens.

Aus der Gegenüberstellung wird deutlich, dass sowohl die verpflichtende als auch die freiwillige Blogaktivität für Studierende mit einem hohen zeitlichen Aufwand verbunden ist. Der Aufwand umfasst, gemäss des «framework of educational affordance of blogs» von Deng und Yuen (2007; 2009b; 2011) folgende Schlüsselaspekte: Lesen, Schreiben, Kommentieren und Kommentare erhalten. Diese Aspekte wiederum sind mit diversen Lernaktivitäten assoziiert (Garcia, Brown, und Elbeltagi 2012):

- Erfahrungen dokumentieren, Gedanken publizieren, Emotionen in multimodaler Form ausdrücken mit Hilfe von Texten, Bildern, Audio- und Videofiles, gehen mit einer Selbstdarstellung einher.

- Strukturieren und Durchdenken von Überlegungen und Ideen erfordert Selbstreflexion.

- Blogposts der Peers und Lehrpersonen zu lesen, führt zu sozialer Verbundenheit und Reflexion.

- Durch das Kommentieren von Blogposts, Verlinken von Weblogs und einzelnen Blogposts entsteht eine soziale Interaktion zwischen Studierenden und Lehrpersonen. 
- Schreiben von Kommentaren zu Blogposts führt zu einem (öffentlichen) Diskurs zwischen Studierenden, Lehrpersonen und Experten im Sinne eines reflexiven Dialoges.

Diese Lernaktivitäten erfordern grundsätzlich die Verschriftlichung von Inhalten bzw. Gedanken, was mit einem hohen zeitlichen Aufwand verbunden ist - und zwar unabhängig von einer verpflichtenden und freiwilligen Blogaktivität. Bei verpflichtendem Bloggen nennen die Studierenden darüber hinaus auch einen inhaltlichen Aufwand. In wie fern dieser inhaltliche Aufwand mit «forced writing» zusammenhängt, ist noch zu prüfen (Deng und Yuen 2009a).

Die extrinsische Motivation der Studierenden ist eine weitere Herausforderung des studentischen Blogeinsatzes. Sim und Hew (2010) verweisen in diesem Zusammenhang auf die Selbstbestimmungstheorie von Ryan und Deci (2000a, 2000b), die postuliert, dass das Verhalten der Studierenden motivational gesteuert ist. Studierende, deren Verhalten eine Reaktion auf externe Faktoren ist, darunter Belohnung oder Zwang, gelten als extrinsisch motiviert. Studierende, die aufgrund innerer Faktoren handeln, z.B. aufgrund eines genuinen Bedürfnisses nach Erkenntnisgewinn, sind intrinsisch motiviert.

Die Ergebnisse der Literaturanalyse lassen vermuten, dass auch im Kontext des freiwilligen Bloggens die extrinsische Motivation stärker ausgeprägt ist als die intrinsische Motivation, zumal Studierende der Aufgabenfokussierung und Ergebnisorientierung ein hohes Gewicht beimessen (Kerawalla et al. 2008). Als Konsequenz könnten Studierende daran gehindert sein, die Grundprinzipien des Bloggens und die damit verbundenen Lernaktivitäten im Sinne einer umfassenden Nutzung zu verwenden. Diese, aber auch die Überlegungen von Dalgarno, Reupert, und Bishop (2015), dass manche Studierende einen inneren Widerstand haben, sich an Blogaktivitäten zu beteiligen, könnte im Zusammenhang mit der Forschung zu Personenmerkmalen und Blogverhalten vertieft untersucht werden (Guadagno, Okdie, und Eno 2008).

Die Ergebnisse zu den Vorgaben sind widersprüchlich. Im Zusammenhang mit verpflichtendem Bloggen wurden Vorgaben sowohl als Herausforderung als auch als Chance genannt. Bei freiwilligem Bloggen wurde das Fehlen von Vorgaben als Herausforderung identifiziert. Gemäss Deng und Yuen (2009a) gibt es keine verbindlichen Regeln zu Vorgaben. Sie betonen, dass sich diese Frage nicht im Sinne von «entweder - oder» beantworten lässt. Diese Aussage spiegelt die im Exkurs dargestellten Ausführungen wider, dass Zwang und Freiheit nicht als binäre Gegensätze verstanden werden können. Die praktischen Implikationen der Autoren könnten als Ansatz verstanden werden, Zwang und Freiheit zu vereinbaren. Aus ihrer Sicht müssen die Vorgaben zur Blogaktivität an die Struktur der Lehrveranstaltung angepasst werden, sowie an den Dialog zwischen den am Lernprozess beteiligten Personen und der Autonomie der Lernenden, basierend auf den Lernzielen, als auch am pädagogischen Bedarf und den Personenmerkmalen der Studierenden. 
Panke, Gaiser, und Maaß (2012) verweisen auf Bloggen als besondere Textform. Bislang fanden sich nur vereinzelte Studien, die die Textform Bloggen vertieft analysierten. Lester und Paulus (2011) konnten anhand ihrer Studie zeigen, dass die Blogaktivität im Hochschulkontext von den Studierenden als «academic talk» wahrgenommen wurde. Erste Analysen ergaben, dass dieser «academic talk» anhand von spezifischen Formulierungen in den Blogposts umgesetzt wird. So benutzten Studierende beispielsweise «I don't know much»-Eingangsformulierungen und «vage Beschreibungen» als Schutzmassnahme im Zusammenhang mit einer akademischen Aufgabe. Weitere Analysen dieser Art sind notwendig, um mehr über die mit der besonderen Textform des Bloggens verbundenen Charakteristika zu erfahren.

Ähnlich wie Deng und Yuen (2011) in ihrem «framework of educational affordance of blogs» entwickelten Kerawalla et al. (2008) ihre Überlegungen zu unterschiedlichen Blog-Verhaltensweisen im Hochschulkontext zu einem «empirically grounded framework» (Kerawalla et al. 2009) weiter. Aus beiden Ansätzen wird deutlich, dass Weblogs als vielseitiges und flexibles Medium mit Diversität einhergehen - hinsichtlich Blog-Verhalten und Lernaktivitäten der Studierenden.

Anhand der beiden Aspekte Schreibfrequenz und persönliche Bedeutsamkeit, lässt sich ebenfalls ein Bezug, zu dem, in den theoretischen Positionen dargelegten Verständnis von Zwang und Freiheit herstellen. Dabei wird insbesondere deutlich, dass Zwang und Freiheit graduell und oft unmerklich ineinander übergehen und einander bedingen. Wie aus den Ergebnissen von Freeman und Brett (2012) deutlich wurde, gelang es den Studierenden erst durch eine Regelmässigkeit und Häufigkeit - im Sinne von Zwang - Weblogs als persönlichen Raum aufzubauen. Persönlich bedeutsame Themen wurden von den Studierenden am ehesten bei freiwilliger Blogaktivität behandelt. Dabei wird ebenso deutlich, dass sich aus mediendidaktischer Perspektive beim verpflichtenden als auch im freiwilligen studentischen Blogeinsatz Ansätze des situierten Lernens (Tulodziecki und Herzig 2004) wiederfinden.

Allerdings wird aus der Literaturanalyse ebenfalls deutlich, dass sich die Forschung zum Blogeinsatz in der Hochschule an der Schnittstelle zu Mediendidaktik und Hochschul(medien-)didaktik auf Forschungsgrundlagen und Theorien aus verschiedenen Disziplinen bezieht.

Die Frage nach den Formen des studentischen Blogeinsatzes wird nachfolgend anhand der Analyse der formalen Merkmale beantwortet.

In der Hochschullehre erfolgt der studentische Blogeinsatz mehrheitlich verpflichtend. Diese Verpflichtung geht mit einer Benotung einher. Dabei zeigen sich zwei Herangehensweisen. Zum einen dient die Häufigkeit der Blogaktivität als Grundlage der Benotung. Studierende müssen dabei eine gewisse Anzahl von Blogposts und Kommentaren verfassen (Xie, Ke, und Sharma 2008; Lester und Paulus 2011). Alternativ wird der Inhalt und die Qualität der Blog-Beiträge anhand vorgegebener 
Beurteilungsraster benotet. Kriterien dafür sind z.B. Vollständigkeit des Blogposts, Sprache, Layout und Darstellung, Anzahl der verwendeten Quellen sowie korrektes Zitieren (Bridges, Harnish, und Sillman 2012; Hodgson und Wong 2011; Lin 2014; Martos-Garcia, Usabiaga, und Valencia-Peris 2017; Robinson-Bryant 2012). Aber auch Kriterien wie die Relevanz der betrachteten Aspekte, Differenziertheit der Analyse und Ableitung konstruktiver und handlungsleitender Verbesserungsmöglichkeiten (Gebhardt und Jenert 2011; Freeman und Brett 2012) werden bewertet. Bei der Vorgabe, eine geringe Anzahl an Blogposts zu verfassen, wird tendenziell eher der Inhalt bzw. die Qualität der Blogposts benotet (Bridges, Harnish, und Sillman 2012).

Obwohl Sim und Hew (2010) in ihrem Literaturreview bereits darauf hinwiesen, finden sich auch in dieser Literaturanalyse nur wenige Artikel bzw. Studien, bei denen die Dauer der Blogtätigkeit über ein Semester hinaus ging (Chu, Chan, und Tiwari 2012; Goktas und Demirel 2012; Lin 2014). Ein möglicher Grund für das Fehlen des längerfristigen Einsatzes von Weblogs könnte sein, dass neben der Forderung nach der didaktischen Verortung von Weblogs in der jeweiligen Lehrveranstaltung (Akbari, Schmidt, und Spannagel 2008) auch eine curriculare Integration des studentischen Blogeinsatzes notwendig wäre (Grell und Rau 2011). Solche Ansätze sind mit einem grossen Aufwand verbunden und fehlen derzeit noch. Auch ist unklar, wie sich ein längerfristiger Einsatz von Weblogs auf die Motivation der Studierenden auswirkt.

Gruppenblogs, bei denen Studierende in Kleingruppen Blogposts und Kommentare verfassen, sind die in der Hochschullehre am häufigsten eingesetzte Sozialform. Begründungen dazu finden sich z.B. bei Halic et al. (2010):

«Blogging provides a means for students to connect in smaller groups to overcome the anonymity of a large lecture hall, working together to create a community of learners» (ebd., 208).

Obwohl sich aus der Analyse der formalen Merkmale des studentischen Bloggens einige Tendenzen im Zusammenhang mit dem freiwilligen oder verpflichtenden Einsatz zeigen, lassen sich diese (noch) nicht statistisch signifikant belegen. Nichtsdestotrotz wäre in einem nächsten Schritt sowohl quantitativ als auch qualitativ zu prüfen, ob sich Zusammenhänge zwischen Verpflichtungsgrad und didaktischen Merkmalen wie zum Beispiel dem Verwendungszweck des Bloggens finden lassen. Hinweise dazu finden sich bei Chu, Chan, und Tiwari (2012). Ausgangspunkt für weitere Studien könnten die von Sim und Hew (2010) beschriebenen Verwendungszwecke und die verschiedenen Lernaktivitäten von Deng und Yuen (2011) sein.

Die hier vorgestellte Literaturanalyse hat Grenzen. Die Suchergebnisse zum freiwilligen Einsatz von Weblogs waren vergleichsweise gering. Aufgrund fehlender Studien für eine Metaanalyse lag der Fokus der Auswertung auf einer qualitativ inhaltlichen Analyse. Ein Grossteil der Aussagen ist das Resultat von Beschreibungen und Auslegungen und weniger das von Berechnungen. Die Ergebnisse der Artikel müssen 
auch kritisch hinterfragt werden, da forschungsmethodologisch in erster Linie gelungene Ansätze veröffentlichtet werden und wenig über allfällige Probleme berichtet wird.

Zusammenfassend konnte gezeigt werden, dass sich der Einsatz von Weblogs in der Hochschullehre durchaus im Verhältnis von Zwang und Freiheit bewegt. Eine systematische Auseinandersetzung mit den Chancen und Herausforderungen, die sich aus dem verpflichtenden und freiwilligen Einsatz ergeben fehlte bislang. Zur Schliessung dieser Lücke trägt der hier vorgestellte Beitrag bei.

Es konnten zum einen konkrete Chancen und Herausforderungen des jeweiligen Einsatzes identifiziert werden. Zum anderen konnten durch die Verdichtung der hier vorgestellten Ergebnisse, einige Bezüge zu theoretischen Positionen hergestellt werden und aufgezeigt werden, wie diese in der Praxis berücksichtigt werden können.

Zwang und Freiheit beim Einsatz von Weblogs in der Hochschullehre verstehen sich nicht als binärer Gegensatz, vielmehr besteht die Möglichkeit diese prozessual aufeinander zu beziehen. Allerdings zeigen die Ergebnisse, dass bei der Verbindung von Zwang und Freiheit eine Vielzahl von Merkmalen zu berücksichtigen sind, die sich nicht allein aus den mediendidaktischen Grundlagen ableiten lassen. Zum einen sind bei der didaktischen Verortung von Weblogs im Unterricht die Struktur der Lehrveranstaltung, die Interaktion und der Dialog zwischen den am Lernprozess beteiligten Personen mit dem pädagogischen Bedarf der Blogaktivität abzustimmen. Personenmerkmale der Studierenden wie z.B. Selbstbestimmung, Motivation und Persönlichkeitsfaktoren haben ebenfalls Einfluss auf die Blogaktivität. Zum anderen erscheint bei einem längerfristigen Einsatz die curriculare Integration des studentischen Bloggens unumgänglich.

\section{Literatur}

Akbari, Mostafa, Tim Schmidt, und Christian Spannagel. 2008. «Ein Planungsraster zum Einsatz von Weblogs in der Lehre». In Workshop Proceedings der Tagungen Mensch \& Computer 2008, DeLFI 2008 und Cognitive Design 2008, herausgegeben von U. Lucke, M. C. Kindsmüller, S. Fischer, \& M. Herczeg, und S. Seehusen, 305-10. Logos. https://dl.gi.de/handle/20.500.12116/7080.

Appelt, Ralf. 2010. «Einsatzpotenziale von (Micro)Blogging in der Weiterbildung». In E-Learning in Hochschule und Weiterbildung: Einsatzchancen und Erfahrungen. Reihe Erwachsenenbildung und lebensbegleitendes Lernen, herausgegeben von Roland Holten und Dieter Nittel, 147-62. Bielefeld: W. Bertelsmann Verlag. https://doi.org/10.3278/6001628w.

Bending, Hazel. 2015. «Blogging my reflection? No I don't but it's a good idea». In Edulearn15: 7th International Conference on Education and New Learning Technologies, herausgegeben von L. Gomez Chova, A. Lopez Martinez, und I. Candel Torres, 3270-77. EDULEARN Proceedings. https://marjon.collections.crest.ac.uk/15738/1/BENDING2015BLO.pdf. 
Blood, Rebecca. 2000. «Weblogs: A history and perspective». rebecca's pocket (blog). 2000. http://www.rebeccablood.net/essays/weblog_history.html.

Bridges, K. Robert, Richard J. Harnish, und Deborah Sillman. 2012. «Teaching undergraduate positive psychology: An active learning approach using student blogs». Psychology Learning \& Teaching 11 (2): 228-37. https://doi.org/10.2304/plat.2012.11.2.228.

Buchem, Ilona, Ralf Appelt, Sascha Kaiser, Sandra Schön, und Martin Ebner. 2011. «Blogging und Microblogging: Anwendungsmöglichkeiten im Bildungskontext». Herausgegeben von Martin Ebner und Sandra Schön. Lehrbuch für Lernen und Lehren mit Technologien. https:// I3t.tugraz.at/index.php/LehrbuchEbner10/article/view/63.

Chu, Samuel K. W., Carol K. K. Chan, und Agnes F. Y. Tiwari. 2012. «Using blogs to support learning during internship». Computers \& Education 58 (3): 989-1000. https://doi.org/10.1016/j. compedu.2011.08.027.

Dalgarno, Barney, Andrea Reupert, und Andrea Bishop. 2015. «Blogging while on professional placement: Explaining the diversity in student attitudes and engagement». Technology, Pedagogy and Education 24 (2): 189-209. https://doi.org/10.1080/1475939X.2013.847481.

Deng, Liping, und Allan H. K. Yuen. 2009a. «Blogs in Higher Education: Implementation and Issues». TechTrends 53 (3): 95-98. http://www.learntechlib.org/p/65596/.

Deng, Liping, und Allan H. K. Yuen. 2009. «Value of Blogs in Preservice Teacher Education». In $17^{\text {th }}$ International Conference on Computers in Education, herausgegeben von S.C. Kong, $\mathrm{H}$. Ogata, H.C. Arnseth, C.K.K. Chan, T. Hirashima, F. Klett, J.H.M. Lee, u. a. Asia-Pacific Society for Computers in Education. http://citeseerx.ist.psu.edu/viewdoc/download?doi=10.1.1.4 83.2626\&rep=rep $1 \&$ type $=$ pdf.

Deng, Liping, und Allan H.K. Yuen. 2007. «Exploring the role of weblogs in supporting learning communities: An integrative approach». In ICT: Providing choices for learners and learning. ascilite, 193-201. http://hdl.handle.net/10722/109628.

Deng, Liping, und Allan H.K. Yuen. 2011. «Towards a framework for educational affordances of blogs». Computers \& Education 56: 441-51. https://doi.org/10.1016/j.compedu.2010.09.005.

Engelhardt, Pia Maria, und Christina Seidl. 2011. «Erziehung zwischen Freiheit und Zwang Zum Problem einer paradoxen Beziehung». In Zeitlose Probleme der Pädagogik-Pädagogik als zeitloses Problem?, herausgegeben von Thomas Mikhal. Karlsruhe: KIT Scientific Publishing. https://doi.org/10.5445/KSP/1000024489.

Fessakis, Georgios, Angelique Dimitracopoulou, und Aggelos Palaiodimos. 2013. «Graphical interaction analysis impact on groups collaborating through blogs». Journal of Educational Technology \& Society 16 (1): 243-53. https://www.j-ets.net/ets/journals/16_1/21.pdf.

Fessakis, Georgios, Konstantinos Tatsis, und Angelique Dimitracopoulou. 2008. «Supporting 〈Learning by Design` Activities Using Group Blogs». Educational Technology \& Society 11 (4): 199-212. https://pdfs.semanticscholar.org/c6fe/87a9c5747edd44be60ea85647a6b0a9 0a4a3.pdf.

Foucault, Michel. 1993. "About the Beginning of the Hermeneutics of the Self. Two Lectures at Dartmouth». Political Theory 21 (2): 198-227. https://doi.org/10.1177/00905917930210 02004. 
Freeman, Wendy, und Clare Brett. 2012. «Prompting authentic blogging practice in an online graduate course». Computers \& Education 59 (3): 1032-41. https://doi.org/10.1016/j.compedu.2012.03.019.

Fulda, Daniel, Hartmut Rosa, und Heinz Thoma, Hrsg. 2018. Freiheit und Zwang: Studien zu ihrer Interdependenz von der Aufklärung bis zur Gegenwart. Paderborn: Wilhelm Fink Verlag.

Garcia, Elaine, Mel Brown, und Ibrahim Elbeltagi. 2012. "The effectiveness of collective group blogs as a tool for reflection within experimental learning projects: A case study of simulated work based learning within higher education». In Inted2012: International Technology, Education and Development Conference, herausgegeben von L. G. Chova, A. L. Martinez, und I. C. Torres, 4676-85. https://library.iated.org/view/GARCIA2012EFF.

Gebhardt, Anja, und Tobias Jenert. 2011. «Besseres Feedback, mehr Reflexion? Fertigkeiten und Einstellungen Studierender zum Bloggen in Praxisprojekten». In Wissensgemeinschaften. Digitale Medien - Öffnung und Offenheit in Forschung und Lehre, herausgegeben von Thomas Köhler und Jörg Neumann, 284-93. Münster; New York; München; Berlin: Waxmann. http://nbn-resolving.de/urn:nbn:de:0111-pedocs-116693.

Giesinger, Johannes. 2011. «Wie kultiviere ich die Freiheit bei dem Zwange? Zu Kants Pädagogik». Pädagogsiche Rundschau 65 (3): 250-70. https://www.academia.edu/28242339/ Wie_kultiviere_ich_die_Freiheit_bei_dem_Zwange_Zu_Kants_P\%C3\%A4dagogik.

Goktas, Yuksel, und Turgay Demirel. 2012. «Blog-enhanced ICT courses: Examining their effects on prospective teachers' ICT competencies and perceptions». Computers \& Education 58 (3): 908-17. https://doi.org/10.1016/j.compedu.2011.11.004.

Grell, Petra, und Franco Rau. 2011. «Partizipationslücken - Social Software in der Hochschullehre». Herausgegeben von Kerstin Mayrberger und Heinz Moser. MedienPädagogik: Zeitschrift für Theorie und Praxis der Medienbildung 21 (Partizipationschancen im Kulturraum Internet nutzen und gestalten: Das Beispiel Web 2.0): 1-23. https://doi.org/10.21240/ mpaed/21/2011.11.21.X.

Guadagno, Rosanna E., Bradley M. Okdie, und Cassie A. Eno. 2008. «Who blogs? Personality predictors of blogging». Computers in Human Behavior 24 (5): 1993-2004. https://doi. org/10.1016/j.chb.2007.09.001.

Halic, Olivia, Debra Lee, Trena Paulus, und Marsha Spence. 2010. «To blog or not to blog: Student perceptions of blog effectiveness for learning in a college-level course». Internet and Higher Education 13 (4): 206-13. https://doi.org/10.1016/j.iheduc.2010.04.001.

Herrera Pavo, Miguel Ángel, und Jesús Casado Rodrigo, und Jesús Casado Rodrigo. 2015. «Interaction analysis of a blog/journal of teaching practice». The Internet and Higher Education 27: 32-43. https://doi.org/10.1016/j.iheduc.2015.05.003.

Hodgson, Paula, und Dora Wong. 2011. «Developing professional skills in journalism through blogs». Assessment \& Evaluation in Higher Education 36 (2): 197-211. https://doi. org/10.1080/02602930903229868.

Jimoyiannis, Athanassios, und Panagiotis Tsiotakis. 2017. «Beyond Students' Perceptions: Investigating Learning Presence in an Educational Blogging Community». Journal of Applied Research in Higher Education 9 (1): 129-46. https://doi.org/10.1108/JARHE-06-2015-0046. 
Jones, Christopher. 2015. Networked Learning. An Educational Paradigm for the Age of Digital Networks. Research in Networked Learning. Cham Heidelberg New York Dordrecht London: Springer Int. Publishing Switzerland. https://doi.org/10.1007/978-3-319-01934-5.

Kant, Immanuel. 2018. «Über Pädagogik». Königsberg. http://www.deutschestextarchiv.de/ kant_paedagogik_1803.

Kerawalla, Lucinda, Shailey Minocha, Gill Kirkup, und Gráinne Conole. 2008. «Characterising the different blogging behaviours of students on an online distance learning course». Learning, Media and Technology 33 (1): 21-33. https://doi.org/10.1080/17439880701868838.

Kerawalla, Lucinda, Shailey Minocha, Gill Kirkup, und Gráinne Conole. 2009. «An empirically grounded framework to guide blogging in higher education». Journal of Computer Assisted Learning 25 (1): 31-42. https://doi.org/10.1111/j.1365-2729.2008.00286.x.

Lester, Jessica N., und Trena M. Paulus. 2011. «Accountability and public displays of knowing in an undergraduate computer-mediated communication context». Discourse Studies 13 (6): 671-86. https://doi.org/10.1177/1461445611421361.

Lin, Ming Huei. 2014. «Effects of Classroom Blogging on ESL Student Writers: An Empirical Reassessment». Asia-Pacific Education Researcher 23 (3): 577-90. https://doi.org/10.1007/ s40299-013-0131-8.

Martos-Garcia, Daniel, Oidui Usabiaga, und Alexandra Valencia-Peris. 2017. «Students' Perception on Formative and Shared Assessment: Connecting Two Universities through the Blogosphere». Journal of New Approaches in Educational Research 6 (1): 64-70. https://doi. org/10.7821/naer.2017.1.194.

Maurer, Susanne, und Susanne Maria Weber. 2006. «Die Kunst, nicht dermaßen regiert zu werden». In Gouvernementalität und Erziehungswissenschaft: Wissen - Macht - Transformation, 9-36. Wiesbaden: VS Verlag für Sozialwissenschaften. https://doi.org/10.1007/978-3531-90194-7_1.

Mazumdar, Pravu. 2015. «Foucault und das Problem der Freiheit». In Foucault und das Problem der Freiheit, herausgegeben von Pravu Mazumdar, 32. Aufl. Staatsdiskurse 32. Stuttgart: Franz Steiner Verlag. http://www.steiner-verlag.de/uploads/tx_crondavtitel/dateidatei/9783515112291_p.pdf.

Missomelius, Petra. 2015. «Bildungsinstitutionen und vernetzte Lernkulturen. Verflechtungen zwischen Ökonomisierung, Öffnung und Molotow-Cocktails». In Medien - Bildung - Dispositive: Beiträge zu einer interdisziplinären Medienbildungsforschung, herausgegeben von Julius Othmer und Andreas Weich, 157-69. Wiesbaden: Springer Fachmedien. https://doi. org/10.1007/978-3-658-07186-8_10.

O’Donnell, Marcus. 2006. «Blogging as pedagogic practice: Artefact and ecology». Asia Pacific Media Educator 17: 5-19. https://ro.uow.edu.au/apme/vol1/iss17/3/. 
Panke, Stefanie, Birgit Gaiser, und Stefanie Maaß. 2012. «Wenn Edusphäre und Blogosphäre sich treffen. Weblogs an Hochschulen zwischen Zitationskartell und Diskursrevolte». Herausgegeben von Kerstin Mayrberger und Heinz Moser. MedienPädagogik: Zeitschrift für Theorie und Praxis der Medienbildung 21 (Partizipationschancen im Kulturraum Internet nutzen und gestalten: Das Beispiel Web 2.0): 1-22. https://doi.org/10.21240/ mpaed/21/2012.03.02.X.

Petticrew, Mark, und Helen Roberts. 2006. Systematic reviews in the social sciences: A practical guide. Malden, MA.: Blackwell Publishing. https://doi.org/10.1002/9780470754887.

Plönges, Sebastian. 2011. «Paradoxien mit E-Portfolios: ein Erfahrungsbericht. Zur Ambivalenz von E-Portfolios in Bildungsprozessen». In Kontrolle und Selbstkontrolle, herausgegeben von Torsten Meyer, Kerstin Mayrberger, Stephan Münte-Goussar, und Christina Schwalbe, 151-53. Wiesbaden: VS Verlag für Sozialwissenschaften. https://doi.org/10.1007/978-3531-92722-0_14.

Riefling, Markus. 2013. Die Kultivierung der Freiheit bei der Macht. Eine pädagogische Betrachtung von Grenzziehung und Grenzüberschreitung. Wiesbaden: Springer Fachmedien. https://doi.org/10.1007/978-3-658-01697-5.

Robinson-Bryant, Frederica. 2012. «Exploring the effects of online blogging on student participation, quality, and the achievement of course outcomes in a freshman engineering course». In 2012 Asee Annual Conference. ASEE Annual Conference \& Exposition. https:// peer.asee.org/21377.

Ryan, Richard M., und Edward L. Deci. 2000a. «Intrinsic and extrinsic motivations: Classic definitions and new directions». Contemporary Education Psychology 25: 54-67. https://doi. org/10.1006/ceps.1999.1020.

Ryan, Richard M., und Edward L. Deci. 2000b. «Self-determination theory and the facilitation of intrinsic motivation, social development, and well-being». American Psychologist 55 (1): 68-78. https://selfdeterminationtheory.org/SDT/documents/2000_RyanDeci_SDT.pdf.

Schindler, Laura A., Gary J. Burkholder, Osama A. Morad, und Craig Marsh. 2017. «ComputerBased Technology and Student Engagement: A Critical Review of the Literature». International Journal of Educational Technology in Higher Education 14 (Oktober): 28. https://doi. org/10.1186/s41239-017-0063-0.

Sharma, Priya, und Philip Tietjen. 2016. «Examining Patterns of Participation and Meaning Making in Student Blogs: A Case Study in Higher Education». American Journal of Distance Education 30 (1): 2-12. https://doi.org/10.1080/08923647.2016.1119605.

Sim, Jeffrey Wee Sing, und Khe Foon Hew. 2010. «The use of weblogs in higher education settings: A review of empirical research». Educational Research Review 5 (2): 151-63. https:// doi.org/10.1016/j.edurev.2010.01.001.

Sullivan, Miriam, und Nancy Longnecker. 2014. "Class blogs as a teaching tool to promote writing and student interaction». Australasian Journal of Educational Technology 30 (4): 390-401. https://doi.org/10.14742/ajet.322. 
Totter, Alexandra, Nives Egger, Felix Schmitz, Thomas Hermann, und Dominik Petko. 2016. «Online-Reflexion mittels Weblogs: Ein Mittel zur Auseinandersetzung mit belastenden Situationen in der berufspraktischen Ausbildung von Lehrpersonen?» Beiträge zur Lehrerinnen- und Lehrerbildung 34 (2): 203-11. http://nbn-resolving.org/urn:nbn:de:0111-pedocs-139432.

Tulodziecki, Gerhard, und Bardo Herzig. 2004. Mediendidaktik. Medien in Lehr- und Lernprozessen. Bd. 2. Handbuch Medienpädagogik. Stuttgart: Klett-Cotta.

Volkers, Achim. 2008. Wissen und Bildung bei Foucault. Aufklärung zwischen Wissenschaft und ethisch-ästhetischen Bildungsprozessen. Wiesbaden: VS Verlag für Sozialwissenschaften. https://doi.org/10.1007/978-3-531-90766-6.

Walker, Jill. 2005. «Blog (Weblog)». In Routledge Encyclopedia of Narrative Theory, herausgegeben von David Herman, Manfred Jahn, und Marie-Laure Ryan, 45. London, New York: Routledge.

Wang, Shu-Ming, Huei-Tse Hou, und Sheng-Yi Wu. 2017. «Analyzing the Knowledge Construction and Cognitive Patterns of Blog-Based Instructional Activities Using Four Frequent Interactive Strategies (Problem Solving, Peer Assessment, Role Playing and Peer Tutoring): A Preliminary Study». Educational Technology Research and Development 65 (2): 301-23. https://doi.org/10.1007/s11423-016-9471-4.

Wolf, Katharina. 2010. «Bridging the distance: The use of blogs as reflective learning tools for placement students». Higher Education Research \& Development 29 (5): 589-602. https:// doi.org/10.1080/07294360.2010.502292.

Xie, Ying, Fengfeng Ke, und Priya Sharma. 2008. "The effect of peer feedback for blogging on college students' reflective learning processes». Internet and Higher Education 11: 18-25. https://doi.org/10.1016/j.iheduc.2007.11.001. 


\section{Anhang: Verzeichnis der empirischen Artikel}

\begin{tabular}{|c|c|}
\hline A1 & $\begin{array}{l}\text { Absalom, Matthew, und Diane De Saint Leger. 2011. «Reflecting on Reflection: Learner } \\
\text { Perceptions of Diaries and Blogs in Tertiary Language Study». Arts \& Humanities } \\
\text { in Higher Education } 10 \text { (2): 189-211. https://doi.org/10.1177/1474022210389141. }\end{array}$ \\
\hline A2 & $\begin{array}{l}\text { Al-Fadda, Hind, und Maha Al-Yahya. 2010. «Using Web Blogs as a Tool to Encoura- } \\
\text { ge Pre-Class Reading, Post-Class Reflections and Collaboration in Higher Educa- } \\
\text { tion». US-China Education Review } 7 \text { (7): 100-106. https://doi.org/10.17265/2161- } \\
623 X / 2010.07 A .010 \text {. }\end{array}$ \\
\hline A3 & $\begin{array}{l}\text { Alqudsi-ghabra, Tagreed M., und Mohammad Al-Bahrani. 2012. «Educational Blog- } \\
\text { ging: The Case of Graduate MLIS Students in Kuwait». Libri } 62 \text { (4): 389-402. htt- } \\
\text { ps://doi.org/10.1515/libri-2012-0030. }\end{array}$ \\
\hline A4 & $\begin{array}{l}\text { Aramo-Immonen, Heli, Salvatore Ammirato, und Jari J. Jussila. 2016. «Blogging as } \\
\text { a Virtual Co-Learning Environment in the International Course Context». In Edu- } \\
\text { learn16: 8th International Conference on Education and New Learning Technolo- } \\
\text { gies, herausgegeben von L. G. Chova, A. L. Martinez, und I. C. Torres, 1383-91. } \\
\text { Valenica: lated-Int Assoc Technology Education \& Development. https://doi. } \\
\text { org/10.21125/edulearn.2016.1275. }\end{array}$ \\
\hline B1 & $\begin{array}{l}\text { Badenhorst, Cecile, und Charles Mather. 2014. «Blogging geographies». Journal of } \\
\text { Geography in Higher Education } 38 \text { (2): 193-207. https://doi.org/10.1080/0309826 } \\
\text { 5.2014.908276. }\end{array}$ \\
\hline B2 & $\begin{array}{l}\text { Bending, Hazel. 2015. «Blogging my reflection? No I don't but it's a good idea». In } \\
\text { EDULEARN Proceedings, herausgegeben von L. Gomez Chova, A. Lopez Martinez, } \\
\text { und I. Candel Torres, 3270-77. https://marjon.collections.crest.ac.uk/15738/1/ } \\
\text { BENDING2015BLO.pdf. }\end{array}$ \\
\hline B3 & $\begin{array}{l}\text { Bernhardt, Thomas, und Karsten D. Wolf. 2012. «Akzeptanz und Nutzungsintensität } \\
\text { von Blogs als Lernmedium in Onlinekursen». In Digitale Medien - Werkzeuge für ex- } \\
\text { zellente Forschung und Lehre, herausgegeben von Gottfried Csanyi, Franz Reichl, } \\
\text { und Andreas Steiner, 141-52. Medien in der Wissenschaft ; 61. Münster u.a.: Wax- } \\
\text { mann. http://nbn-resolving.org/urn:nbn:de:0111-opus-83152. }\end{array}$ \\
\hline B4 & $\begin{array}{l}\text { Bhattacharya, Atanu, und Kiran Chauhan. 2010. «Augmenting Learner Autonomy } \\
\text { through Blogging». ELT Journal } 64 \text { (4): 376-84. https://doi.org/10.1093/elt/ccq002. }\end{array}$ \\
\hline B5 & $\begin{array}{l}\text { Bridges, K. Robert, Richard J. Harnish, und Deborah Sillman. 2012. «Teaching un- } \\
\text { dergraduate positive psychology: An active learning approach using student } \\
\text { blogs». Psychology Learning \& Teaching } 11 \text { (2): 228-37. https://doi.org/10.2304/ } \\
\text { plat.2012.11.2.228. }\end{array}$ \\
\hline C1 & $\begin{array}{l}\text { Caldwell, Helen, und Rebecca Heaton. 2016. «The interdisciplinary use of blogs and } \\
\text { online communities in teacher education». International Journal of Information } \\
\text { and Learning Technology } 33 \text { (3). https://doi.org/10.1108/IJILT-01-2016-0006. }\end{array}$ \\
\hline C2 & $\begin{array}{l}\text { Cameron, Michael. 2012. « Economics with Training Wheels». Using Blogs in Teaching } \\
\text { and Assessing Introductory Economics». The Journal of Economic Education } 43 \\
\text { (4): 397-407. https://hdl.handle.net/10289/6708. }\end{array}$ \\
\hline
\end{tabular}




\begin{tabular}{|c|c|}
\hline C3 & $\begin{array}{l}\text { Carbonell, Marc Romero, Nati Cabera Lanzo, Gergeta Ion, und Elena Cano. } 2012 . \\
\text { «Developing Assessment Practices through the use uf Blogs in Higher Education: } \\
\text { An Innovative Experience in the Open University of Catalonia». In Leveraging } \\
\text { Technology for Learning, herausgegeben von T. Frunzeti, R. Jugureanu, L. Cio- } \\
\text { lan, und C. Radu, 1:300-305. eLearning and Software for Education. https://doi. } \\
\text { org/10.5682/2066-026x-12-048. }\end{array}$ \\
\hline C4 & $\begin{array}{l}\text { Chang, Chi-Cheng, Chaoyun Liang, Kuo-Hung Tseng, und Ju-Shih Tseng. 2014. «Using } \\
\text { e-portfolios to elevate knowledge amassment among university students». Com- } \\
\text { puters \& Education 72: 187-95. https://doi.org/10.1016/j.compedu.2013.10.015. }\end{array}$ \\
\hline C5 & $\begin{array}{l}\text { Chang, Yao-Jen, und Yao-Seng Chang. 2014. «Assessing peer support and usability of } \\
\text { blogging in hybrid learning environments». Interactive Learning Environments } 22 \\
\text { (1):3-17. https://doi.org/10.1080/10494820.2011.619889. }\end{array}$ \\
\hline C6 & $\begin{array}{l}\text { Chou, Chun-Mei. 2011. «Student Teachers Socialization Development by Teaching } \\
\text { Blog: Reflections and Socialization Strategies». Turkish Online Journal of Educa- } \\
\text { tional Technology } 10 \text { (2): 190-201. http://files.eric.ed.gov/fulltext/EJ932238.pdf. }\end{array}$ \\
\hline $\mathrm{C} 7$ & $\begin{array}{l}\text { Chu, Samuel K. W., Carol K. K. Chan, und Agnes F. Y. Tiwari. 2012. «Using blogs to sup- } \\
\text { port learning during internship». Computers \& Education } 58 \text { (3): 989-1000. https:// } \\
\text { doi.org/10.1016/j.compedu.2011.08.027. }\end{array}$ \\
\hline C8 & $\begin{array}{l}\text { Chu, Samuel K. W., Alvin C. M. Kwan, und Peter Warning. 2012. «Blogging for Infor- } \\
\text { mation Management, Learning, and Social Support during Internship». Educa- } \\
\text { tional Technology \& Society } 15 \text { (2): 168-78. http://web.hku.hk/ samchu/docs/ } \\
\text { Chu-2012-Blogging-for-information-management-learning-and-social-support- } \\
\text { during-internship.pdf. }\end{array}$ \\
\hline C9 & $\begin{array}{l}\text { Churchill, Daniel. } 2011 . \text { «Web } 2.0 \text { in education: A study of the explorative use of blogs } \\
\text { with a postgraduate class». Innovations in Education and Teaching International } \\
48 \text { (2): 149-58. https://doi.org/10.1080/14703297.2011.564009. }\end{array}$ \\
\hline C10 & $\begin{array}{l}\text { Clark, Taylorann K., und Thomas H. Paulsen. 2016. «Analyzing Student Teacher Cri- } \\
\text { tical Thinking through Blogs in an Electronic Community of Practice». Journal of } \\
\text { Agricultural Education. } 57 \text { (2): 75-92. https://doi.org/10.5032/jae.2016.02075. }\end{array}$ \\
\hline D1 & $\begin{array}{l}\text { Dalgarno, Barney, Andrea Reupert, und Andrea Bishop. 2015. «Blogging while on } \\
\text { professional placement: Explaining the diversity in student attitudes and enga- } \\
\text { gement». Technology, Pedagogy and Education } 24 \text { (2): 189-209. https://doi.org/10 } \\
.1080 / 1475939 \times .2013 .847481 \text {. }\end{array}$ \\
\hline D2 & $\begin{array}{l}\text { Martínez, Chelo de Andrés. 2012. «Developing metacognition at a distance: Sharing } \\
\text { students' learning strategies on a reflective blog». Computer Assisted Language } \\
\text { Learning } 25 \text { (2): 199-212. https://doi.org/10.1080/09588221.2011.636056. }\end{array}$ \\
\hline D3 & $\begin{array}{l}\text { Deed, Craig, und Anthony Edwards. 2010. «Student Blogging: Implications for Lear- } \\
\text { ning in a Virtual Text-Based Environment». In Technology Enhanced Learning: Qua- } \\
\text { lity of Teaching and Educational Reform, herausgegeben von M. D. Lytras, P. O. } \\
\text { DePablos, D. Avison, J. Sipior, Q. Jin, W. Leal, L. Uden, M. Thomas, S. Cervai, und } \\
\text { D. Horner, 73:18-27. https://doi.org/10.1007/978-3-642-13166-0_3. }\end{array}$ \\
\hline
\end{tabular}




\begin{tabular}{|c|c|}
\hline D4 & $\begin{array}{l}\text { Deed, Craig, und Anthony Edwards. 2011. «Unrestricted student blogging: Implica- } \\
\text { tions for active learning in a virtual text-based environment». Active Learning in } \\
\text { Higher Education } 12 \text { (1): 11-21. https://doi.org/10.1177/1469787410387725. }\end{array}$ \\
\hline D5 & $\begin{array}{l}\text { Dippold, Doris. 2009. «Peer feedback through blogs: Student and teacher perceptions } \\
\text { in an advanced German class». ReCALL: Journal of Eurocall } 21 \text { (1): 18-36. https:// } \\
\text { doi.org/10.1017/S095834400900010X. }\end{array}$ \\
\hline D6 & $\begin{array}{l}\text { Dos, Bulent, und Servet Demir. 2013. «The analysis of the blogs created in a blended } \\
\text { course through the reflective thinking perspective». Educational Sciences: Theory } \\
\text { \& Practice } 13 \text { (2): 1335-44. https://files.eric.ed.gov/fulltext/EJ1017275.pdf. }\end{array}$ \\
\hline D7 & $\begin{array}{l}\text { Duarte, Paulo. } 2015 \text {. «The use of a group blog to actively support learning ac- } \\
\text { tivities». Active Learning in Higher Education } 16 \text { (2): 103-17. https://doi. } \\
\text { org/10.1177/1469787415574051. }\end{array}$ \\
\hline D8 & $\begin{array}{l}\text { Duda, Gintaras, und Katherine Garrett. 2008. «Blogging in the Physics Classroom: A } \\
\text { Research-Based Approach to Shaping Students' Attitudes toward Physics». Ameri- } \\
\text { can Journal of Physics } 76 \text { (11): 1054-66. https://doi.org/10.1119/1.2967707. }\end{array}$ \\
\hline $\mathrm{F} 1$ & $\begin{array}{l}\text { Fessakis, Georgios, Angelique Dimitracopoulou, und Aggelos Palaiodimos. } 2013 . \\
\text { «Graphical interaction analysis impact on groups collaborating through blogs». } \\
\text { Journal of Educational Technology \& Society } 16 \text { (1): 243-53. https://www.j-ets.net/ } \\
\text { ets/journals/16_1/21.pdf. }\end{array}$ \\
\hline $\mathrm{F} 2$ & $\begin{array}{l}\text { Fessakis, Georgios, Konstantinos Tatsis, und Angelique Dimitracopoulou. 2008. «Sup- } \\
\text { porting (Learning by Design` Activities Using Group Blogs». Educational Technolo- } \\
\text { gy \& Society } 11 \text { (4): 199-212. https://pdfs.semanticscholar.org/c6fe/87a9c5747ed } \\
\text { d44be60ea85647a6b0a90a4a3.pdf. }\end{array}$ \\
\hline F3 & $\begin{array}{l}\text { Freeman, Wendy, und Clare Brett. 2012. «Prompting authentic blogging practice in } \\
\text { an online graduate course». Computers \& Education } 59 \text { (3): 1032-41. https://doi. } \\
\text { org/10.1016/j.compedu.2012.03.019. }\end{array}$ \\
\hline $\mathrm{G} 1$ & $\begin{array}{l}\text { Garcia, Elaine, Mel Brown, und Ibrahim Elbeltagi. 2012. «The effectiveness of coll- } \\
\text { ective group blogs as a tool for reflection within experimental learning projects: } \\
\text { A case study of simulated work based learning within higher education». In In- } \\
\text { ted2012: International Technology, Education and Development Conference, he- } \\
\text { rausgegeben von L. G. Chova, A. L. Martinez, und I. C. Torres, 4676-85. https:// } \\
\text { library.iated.org/view/GARCIA2012EFF. }\end{array}$ \\
\hline $\mathrm{G} 2$ & $\begin{array}{l}\text { Garcia, Elaine, Ibrahim Elbeltagi, Mel Brown, und Kerry Dungay. 2014. «The implica- } \\
\text { tions of a connectivist learning blog model and the changing role of teaching and } \\
\text { learning». British Journal of Educational Technology } 46 \text { (4): } 877-94 \text {. https://doi. } \\
\text { org/10.1111/bjet.12184. }\end{array}$ \\
\hline G3 & $\begin{array}{l}\text { Gebhardt, Anja, und Tobias Jenert. 2011. «Besseres Feedback, mehr Reflexion? Fer- } \\
\text { tigkeiten und Einstellungen Studierender zum Bloggen in Praxisprojekten». In } \\
\text { Wissensgemeinschaften. Digitale Medien - Öffnung und Offenheit in Forschung und } \\
\text { Lehre., herausgegeben von Thomas Köhler und Jörg Neumann, 284-93. Münster; } \\
\text { New York; München; Berlin: Waxmann. http://nbn-resolving.de/urn:nbn:de:011- } \\
\text { pedocs-116693. }\end{array}$ \\
\hline
\end{tabular}




\begin{tabular}{|c|c|}
\hline G4 & $\begin{array}{l}\text { Gleaves, Alan, Caroline Walker, und John Grey. 2007. «Using digital and paper diaries } \\
\text { for learning and assessment purposes in higher education: A comparative study } \\
\text { of feasibility and reliability». Assessment \& Evaluation in Higher Education } 32 \text { (6): } \\
631-43 . \text { https://doi.org/10.1080/02602930601117035. }\end{array}$ \\
\hline G5 & $\begin{array}{l}\text { Goktas, Yuksel, und Turgay Demirel. 2012. «Blog-enhanced ICT courses: Examining } \\
\text { their effects on prospective teachers' ICT competencies and perceptions». Compu- } \\
\text { ters \& Education } 58 \text { (3): } 908-17 . \text { https://doi.org/10.1016/j.compedu.2011.11.004. }\end{array}$ \\
\hline $\mathrm{H} 1$ & $\begin{array}{l}\text { Halic, Olivia, Debra Lee, Trena Paulus, und Marsha Spence. 2010. «To blog or not to } \\
\text { blog: Student perceptions of blog effectiveness for learning in a college-level } \\
\text { course». Internet and Higher Education } 13 \text { (4): 206-13. https://doi.org/10.1016/j. } \\
\text { iheduc.2010.04.001. }\end{array}$ \\
\hline $\mathrm{H} 2$ & $\begin{array}{l}\text { Herrera Pavo, Miguel Ángel, und Jesús Casado Rodrigo. 2015. «Interaction analysis of } \\
\text { a blog/journal of teaching practice». The Internet and Higher Education 27: 32-43. } \\
\text { https://doi.org/10.1016/j.iheduc.2015.05.003. }\end{array}$ \\
\hline $\mathrm{H} 3$ & $\begin{array}{l}\text { Hodgson, Paula, und Dora Wong. 2011. «Developing professional skills in journalism } \\
\text { through blogs». Assessment \& Evaluation in Higher Education } 36 \text { (2): 197-211. htt- } \\
\text { ps://doi.org/10.1080/02602930903229868. }\end{array}$ \\
\hline $\mathrm{H} 4$ & $\begin{array}{l}\text { Hourigan, Triona, und Liam Murray. 2010. «Investigating the Emerging Generic Fea- } \\
\text { tures of the Blog Writing Task across Three Discrete Learner Groups at a Higher } \\
\text { Education Institution». Educational Media International } 47 \text { (2): 83-101. https:// } \\
\text { doi.org/10.1080/09523987.2010.492674. }\end{array}$ \\
\hline H5 & $\begin{array}{l}\text { Hramiak, Alison, Helen Boulton, und Brian Irwin. 2009. «Trainee teachers’ use of blogs } \\
\text { as private reflections for professional development». Learning, Media and Techno- } \\
\text { logy } 34 \text { (3): } 259-69 \text {. https://doi.org/10.1080/17439880903141521. }\end{array}$ \\
\hline H6 & $\begin{array}{l}\text { Hsu, Hui-Yin, und Shiangkwei Wang. 2010. «The impact of using blogs on college stu- } \\
\text { dents' reading comprehension and learning motivation». Literacy Research and } \\
\text { Instruction } 50 \text { (1): 68-88. https://doi.org/10.1080/19388070903509177. }\end{array}$ \\
\hline $\mathrm{H} 7$ & $\begin{array}{l}\text { Huang, Tien-Chi, Yueh-Min Huang, und Fu-Yun Yu. 2011. «Cooperative Weblog Lear- } \\
\text { ning in Higher Education: Its Facilitating Effects on Social Interaction, Time Lag, } \\
\text { and Cognitive Load». Educational Technology \& Society } 14 \text { (1): 95-106. https:// } \\
\text { www.j-ets.net/ETS/journals/14_1/9.pdf. }\end{array}$ \\
\hline $\mathrm{H} 8$ & $\begin{array}{l}\text { Hume, Margee. 2012. «Adopting Organisation Learning Theory in the Classroom: } \\
\text { Advancing Learning through the Use of Blogging and Self-Reflection». Inter- } \\
\text { national Journal of Learning and Change } 6 \text { (1): 49-65. https://doi.org/10.1504/ } \\
\text { IJLC.2012.045856. }\end{array}$ \\
\hline I1 & $\begin{array}{l}\text { Ifinedo, Princely. 2016. «Assessing Students' Satisfaction with Blog Usage for Lear- } \\
\text { ning: Focus on Perceived Enjoyment, Knowledge Sharing Experience, and Tech- } \\
\text { nology Acceptance Factors». In , herausgegeben von K. S. Soliman, 2455-66. Int } \\
\text { Business Information Management Assoc-Ibima. https://ibima.org/accepted-pa- } \\
\text { per/assessing-students-satisfaction-blog-usage-learning-focus-perceived-enjoy- } \\
\text { ment-knowledge-sharing-experience-technology-acceptance-factors/. }\end{array}$ \\
\hline
\end{tabular}




\begin{tabular}{|c|c|}
\hline 12 & $\begin{array}{l}\text { Ifinedo, Princely. 2017. «Examining Students' Intention to Continue Using Blogs for } \\
\text { Learning: Perspectives from Technology Acceptance, Motivational, and Social- } \\
\text { Cognitive Frameworks». Computers in Human Behavior } 72 \text { (Juli): 189-99. https:// } \\
\text { doi.org/10.1016/j.chb.2016.12.049. }\end{array}$ \\
\hline 13 & $\begin{array}{l}\text { Ion, Georgeta, Elena Cano, und Nati Cabrera. 2016. «Competency Assessment Tool } \\
\text { (CAT). The Evaluation of an Innovative Competency-Based Assessment Expe- } \\
\text { rience in Higher Education» } 25 \text { (5): } 631-48 \text {. https://doi.org/10.1080/147593 } \\
\text { 9X.2015.1134635. }\end{array}$ \\
\hline J1 & $\begin{array}{l}\text { Jackling, Beverley, Riccardo Natoli, Salina Siddique, und Nick Sciulli. 2015. «Student } \\
\text { attitudes to blogs: A case study of reflective and collaborative learning». Assess- } \\
\text { ment \& Evaluation in Higher Education } 40 \text { (4): 542-56. https://doi.org/10.1080/02 } \\
602938.2014 .931926 .\end{array}$ \\
\hline $\mathrm{J} 2$ & $\begin{array}{l}\text { Jain, Ameeta, Dianne Thomson, Alan Farley, und Pamela Mulready. 2012. "Enga- } \\
\text { gement and learning through social software in finance: A retrospective on the } \\
\text { Trading Room experience». Assessment \& Evaluation in Higher Education } 37 \text { (6): } \\
\text { 701-18. https://doi.org/10.1080/02602938.2011.563280. }\end{array}$ \\
\hline $\mathrm{J} 3$ & $\begin{array}{l}\text { Jenert, Tobias, Anja Gebhardt, und Reto Käser. 2011. «Weblogs zur Unterstützung der } \\
\text { Theorie-Praxis-Integration in der Wirtschaftslehrenden-Ausbildung». Zeitschrift } \\
\text { für E-learning } 6 \text { (2): 17-29. }\end{array}$ \\
\hline $\mathrm{J} 4$ & $\begin{array}{l}\text { Jimoyiannis, Athanassios, und Panagiotis Tsiotakis. 2017. «Beyond Students' Per- } \\
\text { ceptions: Investigating Learning Presence in an Educational Blogging Commu- } \\
\text { nity». Journal of Applied Research in Higher Education } 9 \text { (1): 129-46. https://doi. } \\
\text { org/10.1108/JARHE-06-2015-0046. }\end{array}$ \\
\hline K1 & $\begin{array}{l}\text { Kang, Inae, Curtis J. Bonk, und Myung-Chun Kim. 2011. «A Case Study of Blog-Based } \\
\text { Learning in Korea: Technology becomes Pedagogy». The Internet and Higher Edu- } \\
\text { cation } 14 \text { (4): 227-35. https://doi.org/10.1016/j.iheduc.2011.05.002. }\end{array}$ \\
\hline $\mathrm{K} 2$ & $\begin{array}{l}\text { Kerawalla, Lucinda, Shailey Minocha, Gill Kirkup, und Gráinne Conole. 2008. "Cha- } \\
\text { racterising the different blogging behaviours of students on an online distance } \\
\text { learning course». Learning, Media and Technology } 33 \text { (1): 21-33. https://doi. } \\
\text { org/10.1080/17439880701868838. }\end{array}$ \\
\hline K3 & $\begin{array}{l}\text { Kerawalla, Lucinda, Shailey Minocha, Gill Kirkup, und Gráinne Conole. 2009. «An } \\
\text { empirically grounded framework to guide blogging in higher education». Jour- } \\
\text { nal of Computer Assisted Learning } 25 \text { (1): 31-42. https://doi.org/10.1111/j.1365- } \\
\text { 2729.2008.00286.x. }\end{array}$ \\
\hline K4 & $\begin{array}{l}\text { Ktoridou, Despo, und Elli Doukanari. 2015. «Student Generated Content in Higher } \\
\text { Education Technology-related Blogs». In Proceedings of } 2015 \text { International Con- } \\
\text { ference on Interactive Mobile Communication Technologies and Learning (IMCL), } \\
\text { 289-94. https://doi.org/10.1109/IMCTL.2015.7359605. }\end{array}$ \\
\hline L1 & $\begin{array}{l}\text { Ladyshewsky, Richard K, und Peter Gardner. 2008. «Peer Assisted Learning and } \\
\text { Blogging: A Strategy to Promote Reflective Practice during Clinical Fieldwork». } \\
\text { Australasian Journal of Educational Technology } 24 \text { (3): 241-57. https://doi. } \\
\text { org/10.14742/ajet.1207. }\end{array}$ \\
\hline
\end{tabular}




\begin{tabular}{|c|c|}
\hline L2 & $\begin{array}{l}\text { Lester, Jessica N., und Trena M. Paulus. 2011. «Accountability and public displays of } \\
\text { knowing in an undergraduate computer-mediated communication context». Dis- } \\
\text { course Studies } 13 \text { (6): } 671-86 . \text { https://doi.org/10.1177/1461445611421361. }\end{array}$ \\
\hline L3 & $\begin{array}{l}\text { Lin, Ming Huei. 2014. «Effects of Classroom Blogging on ESL Student Writers: An Empi- } \\
\text { rical Reassessment». Asia-Pacific Education Researcher } 23 \text { (3): 577-90. https://doi. } \\
\text { org/10.1007/s40299-013-0131-8. }\end{array}$ \\
\hline L4 & $\begin{array}{l}\text { Liou, Hsien-Chin, und Zhong-Yan Peng. 2009. «Training Effects on Computer-Me- } \\
\text { diated Peer Review». System } 37 \text { (3): 514-25. https://doi.org/10.1016/j.sys- } \\
\text { tem.2009.01.005. }\end{array}$ \\
\hline L5 & $\begin{array}{l}\text { Liu, Mei-Hui. 2016. «Blending a Class Video Blog to Optimize Student Learning, Outco- } \\
\text { mes in Higher Education». Internet and Higher Education } 30 \text { (Juli): 44-53. https:// } \\
\text { doi.org/10.1016/j.iheduc.2016.03.001. }\end{array}$ \\
\hline M1 & $\begin{array}{l}\text { Machado, Crystal. 2011. «Gender Differences in Student Discourse on Discussion } \\
\text { Board and Blogs: An Instructor's Quest to Create a Level Playing Field in a Hyb- } \\
\text { rid Classroom». Journal of Interactive Online Learning } 10 \text { (1): 36-48. http://www. } \\
\text { ncolr.org/jiol/issues/pdf/10.1.3.pdf. }\end{array}$ \\
\hline M2 & $\begin{array}{l}\text { Martos-Garcia, Daniel, Oidui Usabiaga, und Alexandra Valencia-Peris. 2017. "Stu- } \\
\text { dents' Perception on Formative and Shared Assessment: Connecting Two Univer- } \\
\text { sities through the Blogosphere». Journal of New Approaches in Educational Re- } \\
\text { search } 6 \text { (1): } 64-70 \text {. https://doi.org/10.7821/naer.2017.1.194. }\end{array}$ \\
\hline M3 & $\begin{array}{l}\text { Miyazoe, Terumi, und Terry Anderson. 2011. «Anonymity in blended learning: Who } \\
\text { would you like to be?» Journal of Educational Technology \& Society } 14 \text { (2): 175-87. } \\
\text { https://www.j-ets.net/ETS/journals/14_2/15.pdf. }\end{array}$ \\
\hline M4 & $\begin{array}{l}\text { Mohamad, Maslawati, Faridah Musa, Maryam Mohamed Amin, Norlaila Mufti, Rozmel } \\
\text { Abdul Latiff, und Nani Rahayu Sallihuddin. 2014. «Improving undergraduates' } \\
\text { critique via computer mediated communication». TOJET: The Turkish Online Jour- } \\
\text { nal of Educational Technology } 13 \text { (3): 88-95. https://files.eric.ed.gov/fulltext/ } \\
\text { EJ1034244.pdf. }\end{array}$ \\
\hline N1 & $\begin{array}{l}\text { Neira-Pineiro, Maria del Rosario. 2015. «Reading and writing about literature on the } \\
\text { Internet. Two innovative experiences with blogs in higher education». Innovations } \\
\text { in Education and Teaching International } 52 \text { (5): 546-57. https://doi.org/10.1080/1 } \\
4703297.2014 .900452 \text {. }\end{array}$ \\
\hline N2 & $\begin{array}{l}\text { Nisiforou, Efi A., und Nikleia Eteokleous. 2014. «The role of blogging in a changing } \\
\text { society: Theory, practice, and implications». In Cases on teacher identity, diver- } \\
\text { sity, and cognition in higher education., herausgegeben von Paul Breen, 342-70. } \\
\text { Advances in higher education and professional development (AHEPD). Hershey, } \\
\text { PA, US: Information Science Reference/IGI Global. https://doi.org/10.4018/978-1- } \\
\text { 4666-5990-2.ch014. }\end{array}$ \\
\hline
\end{tabular}




\begin{tabular}{|c|c|}
\hline N3 & $\begin{array}{l}\text { Nisiforou, Efi A., und Nikleia Eteokleous. 2013. «Defining evaluation criteria in blog- } \\
\text { ging and non blogging: A case study from a pedagogical perspective». In } 7 \text { th Inter- } \\
\text { national Technology, Education and Development Conference, herausgegeben von } \\
\text { L. G. Chova, A. L. Martinez, und I. C. Torres, 4750-58. INTED Proceedings. https:// } \\
\text { library.iated.org/view/NISIFOROU2013DEF. }\end{array}$ \\
\hline N4 & $\begin{array}{l}\text { Novakovich, Jeanette. 2016. «Fostering critical thinking and reflection through blog- } \\
\text { mediated peer feedback». Journal of Computer Assisted Learning } 32 \text { (1): 16-30. } \\
\text { https://doi.org/10.1111/jcal.12114. }\end{array}$ \\
\hline $\mathrm{O} 1$ & $\begin{array}{l}\text { Olofsson, Anders D., J. Ola Lindberg, und Trond Eiliv Hauge. 2011. «Blogs and the } \\
\text { Design of Reflective Peer-to-Peer Technology-Enhanced Learning and For- } \\
\text { mative Assessment». Campus-Wide Information Systems 28. https://doi. } \\
\text { org/10.1108/10650741111145715. }\end{array}$ \\
\hline $\mathrm{O} 2$ & $\begin{array}{l}\text { Osman, Gihan, und Joyce Hwee Ling Koh. 2013. "Understanding management stu- } \\
\text { dents' reflective practice through blogging». The Internet and Higher Education } \\
\text { 16: } 23-31 \text {. https://doi.org/10.1016/j.iheduc.2012.07.001. }\end{array}$ \\
\hline P1 & $\begin{array}{l}\text { Papastergiou, Marina, Vassilis Gerodimos, und Panagiotis Antoniou. 2011. «Multime- } \\
\text { dia blogging in physical education: Effects on student knowledge and ICT self- } \\
\text { efficacy». Computers \& Education } 57 \text { (3): 1998-2010. https://doi.org/10.1016/j. } \\
\text { compedu.2011.05.006. }\end{array}$ \\
\hline P2 & $\begin{array}{l}\text { Park, Ji Yong. 2015. «Student interactivity and teacher participation: An application } \\
\text { of legitimate peripheral participation in higher education online learning environ- } \\
\text { ments». Technology, Pedagogy and Education } 24 \text { (3): 389-406. https://doi.org/10.1 } \\
\text { 080/1475939X.2014.935743. }\end{array}$ \\
\hline P3 & $\begin{array}{l}\text { Philip, Robyn, und J.ennifer Nicholls. 2009. «Group blogs: Documenting collaborative } \\
\text { drama processes». Australasian Journal of Educational Technology } 25 \text { (5): 683-99. } \\
\text { https://doi.org/10.14742/ajet.1115. }\end{array}$ \\
\hline P4 & $\begin{array}{l}\text { Pinya, Carme, und Maria Rosa Rosselló. 2016. «Using Blogs to Be Aware of the Deve- } \\
\text { lopment and Adoption of Professional Skills». Education and Information Techno- } \\
\text { logies } 21 \text { (5): 1055-70. https://doi.org/10.1007/s10639-014-9367-z. }\end{array}$ \\
\hline $\mathrm{R} 1$ & $\begin{array}{l}\text { Rebmann, Kristen Radsliff, und Camden Bernhard Clark. 2017. «Open Access Research } \\
\text { Via Collaborative Educational Blogging: A Case Study from Library \& Information } \\
\text { Science». Open Praxis } 9 \text { (3): 345-57. https://doi.org/10.5944/openpraxis.9.3.665. }\end{array}$ \\
\hline R2 & $\begin{array}{l}\text { Richter, Christoph, Christian Vogel, und Eva Zöserl. 2007. «Mehr als ein Praktikums- } \\
\text { bericht - Konzeption und Evaluation eines Szenarios zur Förderung individueller } \\
\text { und kollektiver Reflexion im Berufspraktikum». In Studieren neu erfinden - Hoch- } \\
\text { schule neu denken., herausgegeben von Marianne Merkt, Kerstin Mayrberger, Rolf } \\
\text { Schulmeister, Angela Sommer, und Ivo van den Berk, 391-400. Münster u.a.: Wax- } \\
\text { mann. http://nbn-resolving.de/urn:nbn:de:0111-pedocs-113769. }\end{array}$ \\
\hline R3 & $\begin{array}{l}\text { Robertson, Judy. 2011. «The educational affordances of blogs for self-directed lear- } \\
\text { ning». Computers \& Education } 57 \text { (2): 1628-44. https://doi.org/10.1016/j.compe- } \\
\text { du.2011.03.003. }\end{array}$ \\
\hline
\end{tabular}




\begin{tabular}{|c|c|}
\hline R4 & $\begin{array}{l}\text { Robinson-Bryant, Frederica. 2012. «Exploring the effects of online blogging on stu- } \\
\text { dent participation, quality, and the achievement of course outcomes in a fresh- } \\
\text { man engineering course». In . ASEE Annual Conference \& Exposition. https://peer. } \\
\text { asee.org/21377. }\end{array}$ \\
\hline R5 & $\begin{array}{l}\text { Rosselló, Maria, und Carme Pinya. 2017. «Using Blogs to Improve Professional Com- } \\
\text { petencies among Undergraduate Students». Digital Education Review 31: 20-38. } \\
\text { http://revistes.ub.edu/index.php/der/article/view/15910. }\end{array}$ \\
\hline S1 & $\begin{array}{l}\text { Sharma, Priya, und Philip Tietjen. 2016. «Examining Patterns of Participation and } \\
\text { Meaning Making in Student Blogs: A Case Study in Higher Education». American } \\
\text { Journal of Distance Education } 30 \text { (1): } 2-12 \text {. https://doi.org/10.1080/08923647.20 } \\
\text { 16.1119605. }\end{array}$ \\
\hline S2 & $\begin{array}{l}\text { Stoszkowski, John, und Dave Collins. 2017. «Using Shared Online Blogs to Structure } \\
\text { and Support Informal Coach Learningpart 1: A Tool to Promote Reflection and } \\
\text { Communities of Practice». Sport Education and Society } 22 \text { (2): 247-70. https://doi. } \\
\text { org/10.1080/13573322.2015.1019447. }\end{array}$ \\
\hline S3 & $\begin{array}{l}\text { Sullivan, Miriam, und Nancy Longnecker. 2014. «Class blogs as a teaching tool to pro- } \\
\text { mote writing and student interaction». Australasian Journal of Educational Tech- } \\
\text { nology } 30 \text { (4): } 390-401 . \text { https://doi.org/10.14742/ajet.322. }\end{array}$ \\
\hline S4 & $\begin{array}{l}\text { Sun, Yu-Chih. 2010. «Developing reflective cyber communities in the blogosphere: } \\
\text { A case study in Taiwan higher education». Teaching in Higher Education } 15 \text { (4): } \\
\text { 369-81. https://doi.org/10.1080/13562510903556075. }\end{array}$ \\
\hline $\mathrm{T} 1$ & $\begin{array}{l}\text { Tekinarslan, Erkan. 2008. «Blogs: A Qualitative Investigation into an Instructor and } \\
\text { Undergraduate Students' Experiences». Australasian Journal of Educational Tech- } \\
\text { nology } 24 \text { (4): 402-12. https://doi.org/10.14742/ajet.1200. }\end{array}$ \\
\hline T2 & $\begin{array}{l}\text { Thoms, Brian. 2011. «A Dynamic Social Feedback System to Support Learning and So- } \\
\text { cial Interaction in Higher Education». leee Transactions on Learning Technologies } \\
4 \text { (4): 340-52. https://doi.org/10.1109/TLT.2011.9. }\end{array}$ \\
\hline T3 & $\begin{array}{l}\text { Timotheou, Maria Mama. 2014. «Reflective Thinking: Exploring Blog Use by Adult } \\
\text { Learners». In Learning and Collaboration Technologies: Technology-Rich Environ- } \\
\text { ments for Learning and Collaboration LTC 2014, herausgegeben von Panayiotis } \\
\text { Zaphiris und Andri Ioannou, 8524:460-66. Lecture Notes in Computer Science. } \\
\text { Cham: Springer. https://doi.org/10.1007/978-3-319-07485-6_45. }\end{array}$ \\
\hline T4 & $\begin{array}{l}\text { Top, Ercan. 2012. «Blogging as a Social Medium in Undergraduate Courses: Sense of } \\
\text { Community Best Predictor of Perceived Learning». Internet and Higher Education } \\
15 \text { (1): } 24-28 . \text { https://doi.org/10.1016/j.iheduc.2011.02.001. }\end{array}$ \\
\hline T5 & $\begin{array}{l}\text { Top, Ercan, Erman Yukselturk, und Fethi A. Inan. 2010. «Reconsidering Usage of Blog- } \\
\text { ging in Preservice Teacher Education Courses». Internet and Higher Education } 13 \\
\text { (4): } 214-17 . \text { https://doi.org/10.1016/j.iheduc.2010.05.003. }\end{array}$ \\
\hline
\end{tabular}




\begin{tabular}{|c|c|}
\hline T6 & $\begin{array}{l}\text { Totter, Alexandra, Nives Egger, Felix Schmitz, Thomas Hermann, und Dominik Pet- } \\
\text { ko. 2016. «Online-Reflexion mittels Weblogs: Ein Mittel zur Auseinandersetzung } \\
\text { mit belastenden Situationen in der berufspraktischen Ausbildung von Lehrper- } \\
\text { sonen?» Beiträge zur Lehrerinnen- und Lehrerbildung } 34 \text { (2): 203-11. http://nbn- } \\
\text { resolving.org/urn:nbn:de:0111-pedocs-139432. }\end{array}$ \\
\hline W1 & $\begin{array}{l}\text { Wang, Shu-Ming, Huei-Tse Hou, und Sheng-Yi Wu. 2017. «Analyzing the Knowledge } \\
\text { Construction and Cognitive Patterns of Blog-Based Instructional Activities } \\
\text { Using Four Frequent Interactive Strategies (Problem Solving, Peer Assessment, } \\
\text { Role Playing and Peer Tutoring): A Preliminary Study». Educational Technology } \\
\text { Research and Development } 65 \text { (2): 301-23. https://doi.org/10.1007/s11423-016- } \\
\text { 9471-4. }\end{array}$ \\
\hline W2 & $\begin{array}{l}\text { Wang, Yi-Shun, Hsien-Ta Li, Ci-Rong Li, und Chian Wang. 2014. «A model for assessing } \\
\text { blog-based learning systems success». Online Information Review } 38 \text { (7): 969-90. } \\
\text { https://doi.org/10.1108/OIR-04-2014-0097. }\end{array}$ \\
\hline W3 & $\begin{array}{l}\text { Wilson, Anna, Susan Howitt, und Denise Higgins. 2016. «Assessing the unassessable: } \\
\text { Making learning visible in undergraduates' experiences of scientific research». As- } \\
\text { sessment \& Evaluation in Higher Education } 41 \text { (6): 901-16. https://doi.org/10.1080 } \\
\text { /02602938.2015.1050582. }\end{array}$ \\
\hline W4 & $\begin{array}{l}\text { Wolf, Katharina. 2010. «Bridging the distance: The use of blogs as reflective learning } \\
\text { tools for placement students». Higher Education Research \& Development } 29 \text { (5): } \\
\text { 589-602. https://doi.org/10.1080/07294360.2010.502292. }\end{array}$ \\
\hline X1 & $\begin{array}{l}\text { Xie, Ying, Fengfeng Ke, und Priya Sharma. 2008. «The effect of peer feedback for blog- } \\
\text { ging on college students' reflective learning processes». Internet and Higher Edu- } \\
\text { cation 11: 18-25. https://doi.org/10.1016/j.iheduc.2007.11.001. }\end{array}$ \\
\hline Y1 & $\begin{array}{l}\text { Yang, Chyan, und Yao-Seng Chang. 2012. «Assessing the effects of interactive blogging } \\
\text { on student attitudes towards peer interaction, learning motivation, and academic } \\
\text { achievements». Journal of Computer Assisted Learning } 28 \text { (2): 126-35. https://doi. } \\
\text { org/10.1111/j.1365-2729.2011.00423.x. }\end{array}$ \\
\hline
\end{tabular}

